\title{
Polyglycerol-based hydrogels and nanogels: from synthesis to applications
}

\author{
Meena Kumarił,2, Suchita Prasad ${ }^{\ddagger} 3$, Ljiljana Fruk*,1 (iD \& Badri Parshad**,1 (iD \\ ${ }^{1}$ Department of Chemical Engineering \& Biotechnology, University of Cambridge, Cambridge, CB3 OAS, UK \\ ${ }^{2}$ Department of Chemistry, Government College for Women, Badhra, Ch. Dadri, Haryana 127308, India \\ ${ }^{3}$ Department of Chemistry, University of Delhi, Delhi 110007, India \\ *Author for correspondence: If389@cam.ac.uk \\ **Author for correspondence: bp448@cam.ac.uk \\ ${ }_{\ddagger}^{\ddagger}$ Authors contributed equally
}

Hydrogels and nanogels have emerged as promising materials for biomedical applications owing to their large surface area and tunable mechanical and chemical properties. Their large surface area is well suited for bioconjugation, whilst the interior porous network can be utilized for the transport of valuable biomolecules. The use of biocompatible hydrophilic building blocks/linkers for the preparation of hydrogels and nanogels not only avoids undesired side effects within the biological system, but also retains high water content, thereby creating an environment which is very similar to extracellular matrix. Their tunable multivalency and hydrophilicity and excellent biocompatibility, together with ease of functionalization, makes polyglycerol macromonomers well suited for synthesizing cross-linked networks that can be used as extracellular matrix mimics. Here we provide an overview of the synthesis of polyglycerol-based hydrogels and nanogels for various biomedical applications.

First draft submitted: 25 June 2020; Accepted for publication: 1 December 2020; Published online:

6 January 2021

Keywords: chemical cross-linking • hydrogel $\bullet$ nanogel $\bullet$ polyglycerol

Hydrogels are 3D cross-linked networks capable of retaining a large amount of water. Due to the possibility to tune their biocompatibility, molecular flexibility, stiffness and binding affinity for biomolecules by various strategies, they have been widely explored as mimics of extracellular matrix. Such matrix is a heterogeneous structure unique to each tissue that contains the physical, biochemical and biomechanical components needed for cell proliferation and communication. The development of extracellular matrices with the desired properties is one of the most important steps in tissue engineering and has been benefited largely from advances in the design of different classes of cross-linked hydrogels [1]. Besides tissue engineering, hydrogels have made a significant contribution in the fields of drug delivery, pathogen inhibition, biomolecule immobilization and the production of hygiene products, wound dressings and contact lenses [2]. The conjugation of suitable ligands on the surface of cross-linked networks further facilitates the delivery of encapsulated guests to the target site as well as improves their affinity to bind with specific pathogens.

The current field of hydrogel research commenced with the preparation of a polyhydroxyethylmethacrylate (pHEMA) hydrogel by Wichterle and Lim in 1960, when these materials were utilized for contact lens applications [3]. The first nanogel was prepared three decades later by self-aggregation of cholesterol-bearing polysaccharides in water that resulted in physically cross-linked nanosized structures [4]. Nanogels are fabricated using various methodologies, such as inverse miniemulsion, inverse nanoprecipitation and microfluidics, and with various crosslinking species. They have received significant attention in recent years owing to their nanosized features and easily tailored chemical and physical properties and have been used as space-filling agents, delivery vehicles for bioactive molecules [5-8], biosensors [9-12], bioimaging [13-16] and antifouling agents [17,18], and also as tissue engineering elements [19-22].

Several excellent review articles have been published concerning hydrogel and nanogel design [23-26], but here we will focus on polyglycerol (PG)-based systems, which have been extensively explored in the past decade. 


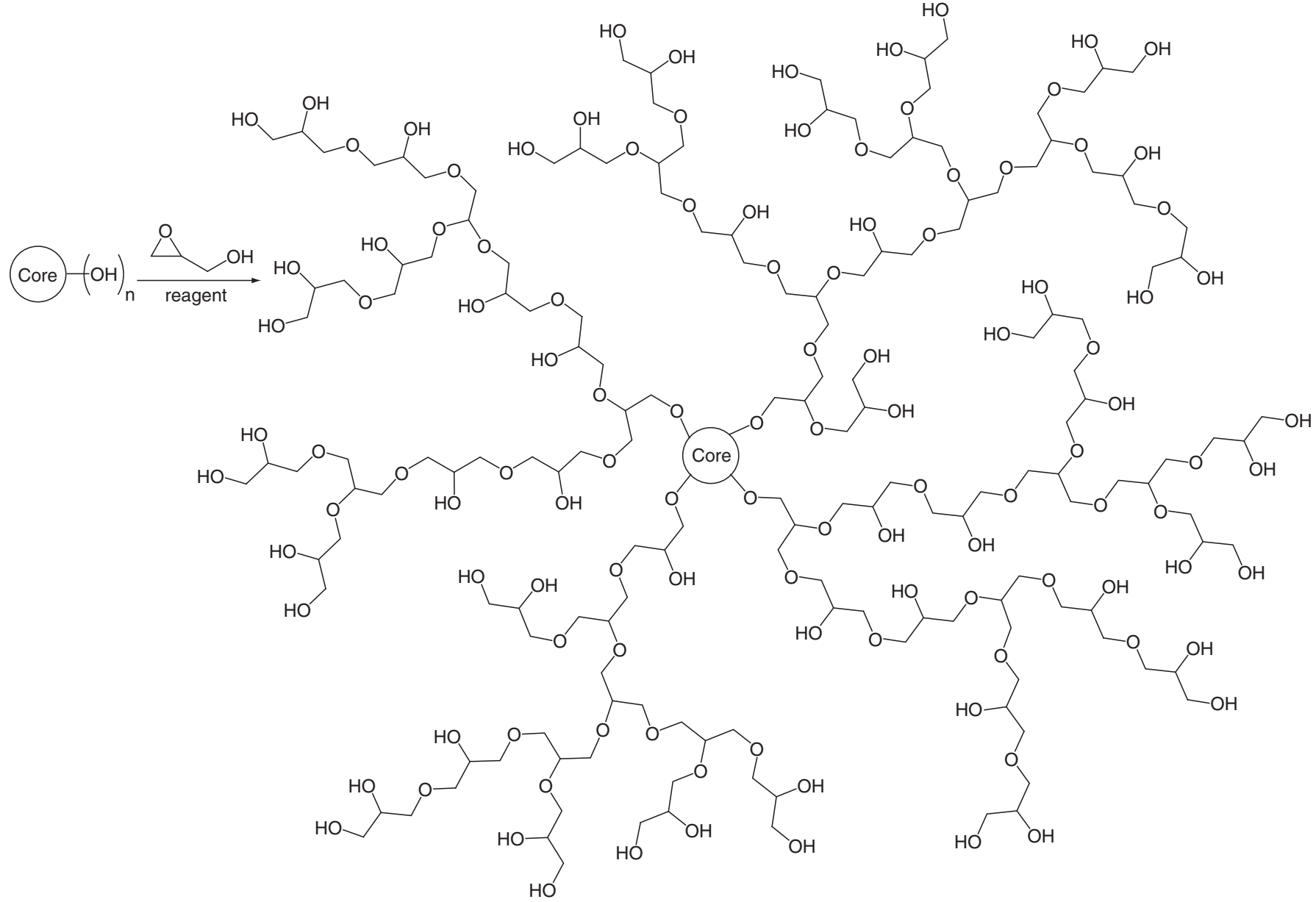

Hyperbranched polyglycerol (hPG) or dendritic polyglycerol (dPG)

Figure 1. General synthesis of linear and hyperbranched/dendritic polyglycerols.

Linear (lPG), hyperbranched PG (hPG) and dendritic PG (dPG) (Figure 1) were used to prepare biocompatible hydrogels and nanogels via enzyme-mediated cross-linking, click chemistry approaches (copper-catalyzed azidealkyne cycloaddition [CuAAC], strain-promoted azide-alkyne cycloaddition [SPAAC] and thiol-ene and thioldisulfide cross-linking), boronic acid-diol based cross-linking, acid-mediated cross-linking and inverse electron demand Diels-Alder (IEDDA) cycloaddition cross-linking strategies. The presence of multiple anchoring points on PG's surface allows for the attachment of different cross-linkers as well as functionalization with other bioactive moieties. These cross-linked architectures have been well characterized in terms of their morphology, swelling behavior, cytocompatibility and rheological and mechanical properties such as viscosity and elasticity, and have been used mainly in tissue repair, drug delivery and sensing applications. In this context, we will discuss various synthetic approaches devised for hydrogel and nanogel formulations, together with their diverse biomedical applications.

\section{Synthetic methods to prepare PG hydrogels \& nanogels}

PG hydrogels and nanogels have been synthesized from PG monomers using a wide range of physical or chemical cross-linking strategies. Among the physical cross-linking interactions, hydrogen bonding, electrostatic interactions involving various ions, van der Waal forces and host-guest interactions have been the most widely employed for gel preparation [27,28]. However, covalently cross-linked hydrogels are often desirable for use in drug delivery, pathogen inhibition and tissue engineering because they have superior mechanical properties and well controllable structures. Most interesting methods used in the preparation of PG gel networks involve the polymerization of bi- or multi-functional macromonomers and their covalent cross-linking employing enzyme and various click chemistry strategies. 


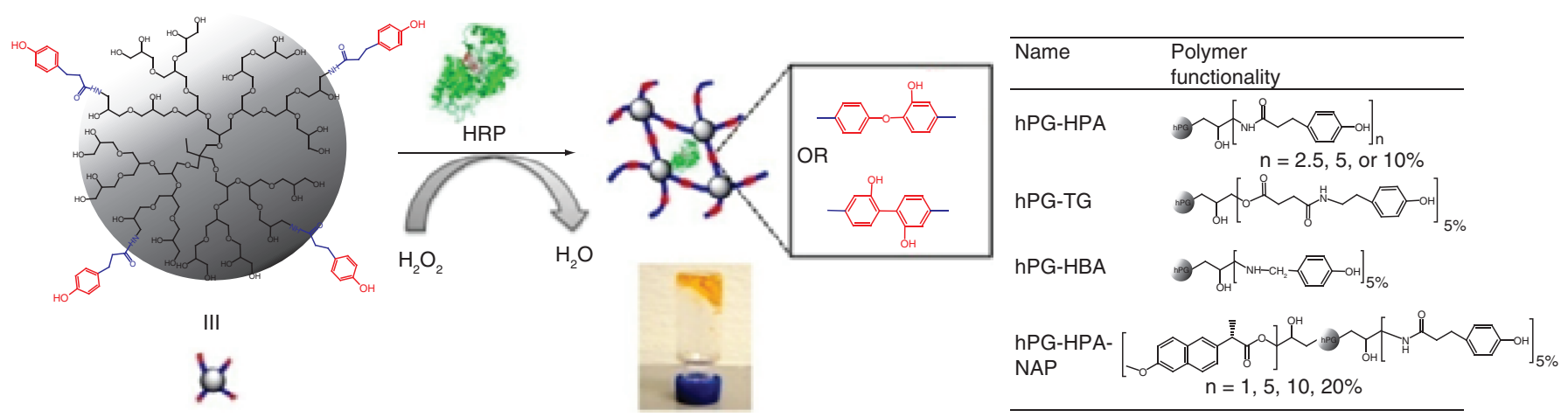

Figure 2. Formation of polyglycerol-HPA hydrogels by enzymatic cross-linking using horseradish peroxidase of polyglycerol monomers modified with horseradish peroxidase substrates.

Reproduced with permission from [29] ๔ American Chemical Society (2014).

\section{Enzyme-mediated cross-linking for preparation of PG hydrogels \& nanogels}

Enzymatic cross-linking provides a unique and efficient green approach for in situ hydrogel and nanogel formation. Unlike conventional physical and chemical cross-linking, enzymatic cross-linking constructs gels with limited side reactions and undesired products due to the high selectivity and specificity of the enzymes. Moreover, enzymatic methods work well under physiological conditions by improving the biological activity of the encapsulated/conjugated guests.

Wu et al. studied horseradish peroxidase (HRP)-mediated cross-linking of dendritic PG polymers and evaluated the influence of temperature and inhibitors on the enzyme-mediated cross-linking (Figure 2) [29]. hPG was first functionalized with a phenol group containing moieties such as 3-(4-hydroxyphenyl) propionic acid (HPA), tyramine and 4-hydroxybenzaldehyde. The HRP-mediated oxidative cross-linking of these linkers with PG in the presence of $\mathrm{H}_{2} \mathrm{O}_{2}$ resulted in tunable hydrogel formation for hPG-HPA and hPG-tyramine, which are wellrecognized HRP substrates [30-32].

In the same study, the glucose-triggered gel formation was also achieved in the presence of glucose oxidase and was found to depend on the glucose concentration, the glucose oxidase or HRP concentration and the temperature, as well as the presence of inhibitors $\left(\mathrm{NaN}_{3}, \mathrm{CuSO}_{4}\right.$ and $\left.\mathrm{AgNO}_{3}\right)$, allowing for better control of the gelation time. Furthermore, high cytocompatibility of these hydrogels against murine L929 fibroblast cell lines supported the use of these gels for wound healing applications.

In another study, Wu et al. presented a similar enzymatic strategy for nanogelation to produce stable 200-nm dPG nanogel particles under physiological conditions for the encapsulation of proteins [33]. This approach utilized HRP-catalyzed oxidative cross-linking of dPG-HPA in the presence of $\mathrm{H}_{2} \mathrm{O}_{2}$ in a water-in-oil inverse miniemulsion. Prepared nanogels were then used to encapsulate the Candida antarctica lipase B enzyme. The fabrication method offered mild cross-linking conditions, a facile nanogel preparation, efficient protein loading and the preservation of the enzyme activity within the scaffold, indicating the huge potential of this strategy for encapsulation of various industrially relevant enzymes.

In another study, Zabihi et al. devised a straightforward synthetic method for the production of biodegradable PG nanoarchitectures using Novozym ${ }^{\circledR} 435$ [34]. Using this enzyme a copolymerization of glycidol and succinic anhydride was achieved, resulting in the formation of poly(glycerol-co-succinic acid) nanogels (Figure 3). Herein, Novozym has played a dual role: it catalyzed the ring-opening copolymerization of glycidol and succinic anhydride, and the esterification of copolymerization products, resulting in covalently cross-linked polymeric networks (nanogels) containing succinic acid in their backbone. While the succinic acid segments offered improved biodegradability and loading capacity of the nanogels, the PG units improved their water solubility, loading capacity and biocompatibility. This was demonstrated by loading of the immunosuppressive drug tacrolimus and a photosensitizer, 5,10,15,20-tetrakis(3-hydroxyphenyl)porphyrin, within the nanogels, and their subsequent use for efficient delivery of the therapeutic cargo to skin epidermis. In addition to enzymatic copolymerization, the authors also explored a thermal and chemical copolymerization strategy using $\mathrm{Sn}(\mathrm{Oct})_{2}$ followed by Novozym $\mathbb{R}$ 435 esterification. 


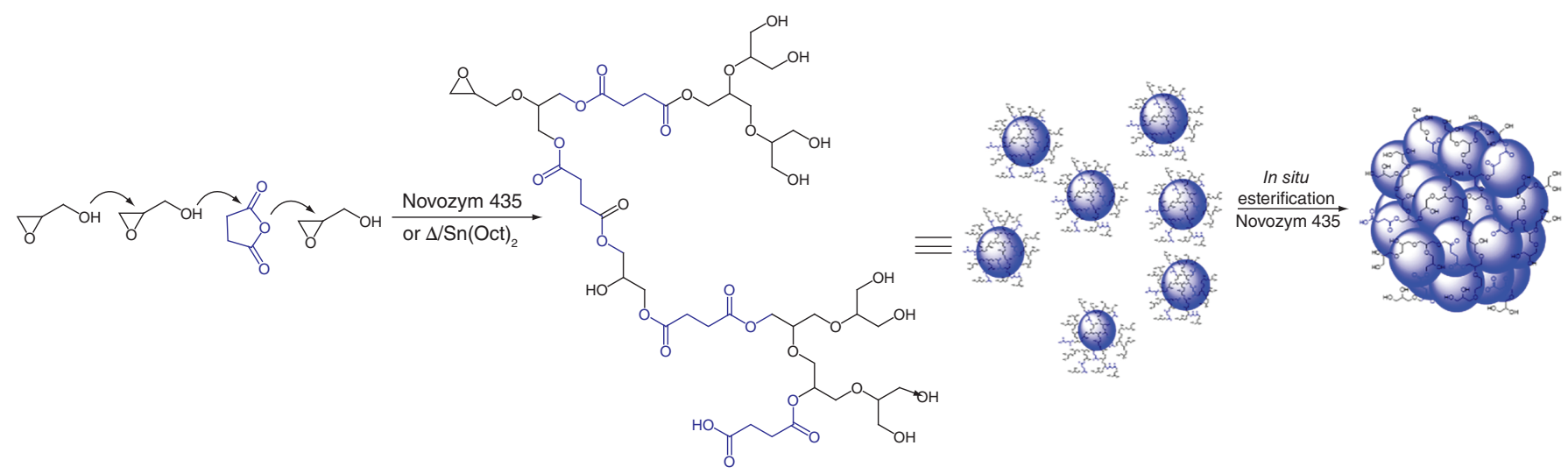

Figure 3. Enzymatic (Novozym $\left.{ }^{\circledR} 435\right)$, thermal $(\Delta)$ and chemical (Sn(Oct) 2 ) catalysis to obtain poly(glycerol-co-succinic) acid, and in situ nanogel formation using enzyme-mediated esterification Modified with permission from [34] (c) American Chemical Society (2019).

More enzyme classes have been employed to prepare various hydrogels using other polymeric monomers and polymer-peptide hybrids, but little has been done in terms of PG cross-linking, indicating a clear niche which should be further explored [35].

\section{Click chemistry strategies for PG gel formation}

Click chemistry denotes a group of reactions that are fast, usually require mild reaction conditions and no catalysts, have high yields of easily isolated products and result in few or no side products. Although a large number of reactions fulfill the criteria, in this review we will focus on azide-alkyne cycloaddition (both $\mathrm{Cu}$-catalyzed and $\mathrm{Cu}$-free) and thiol-ene click reactions, which have emerged as promising tools for hydrogel and nanogel preparations.

\section{Copper-catalyzed azide-alkyne cycloaddition-mediated synthesis of nanogels}

Steinhilber et al. devised a copper-catalyzed azide-alkyne cycloaddition (CuAAC) approach to synthesize biodegradable PG nanogels on different length scales (Figure 4) [36]. dPG was employed as nanogel scaffold material and modified to contain $\mathrm{pH}$-labile benzacetal bonds, which conferred biodegradability. Inverse nanoprecipitation in the absence of surfactant, in combination with the copper-mediated chemical cross-linking of the precipitated dPG bearing propargylated benzacetal groups and an azide-containing dPG $\left(\mathrm{dPG}-\mathrm{N}_{3}\right)$, resulted in PG nanogels $(100-1000 \mathrm{~nm})$. The acidic environment present in inflamed and tumor tissues as well as within the intracellular acidic compartments degraded these nanogels into smaller fragments, ensuring $\mathrm{pH}$-driven degradation. Such a mild approach used to afford biodegradable PG nanogels also permitted the encapsulation of labile biomacromolecules such as proteins (BSA) and the therapeutically relevant enzyme asparaginase, used to treat Hodgkin lymphoma and acute lymphoblastic leukemia.

Another strategy to obtain PG-based multifunctional nanogels was reported by Richter $e$ t al. and is based on the use of IPG and dPG monomers (IPG-dPG nanogels) [37]. This approach employed linear PG bisazide (IPG$\mathrm{Az}_{2}$ ) and dPG functionalized with eight alkyne moieties (dPG-Alk ${ }_{8}$ ) to prepare IPG-dPG nanogels by an inverse nanoprecipitation technique. Such nanogels were $\mathrm{pH}$-responsive, as demonstrated by real-time studies using atomic force microscopy under ambient and in liquid conditions.

\section{Strain-promoted azide-alkyne cycloaddition-mediated synthesis}

Strain-promoted azide-alkyne cycloaddition (SPAAC) click reactions have advantages over other methods of crosslinking polymerization as they occur under physiological conditions and neither require any external triggers nor leave any harmful residual by-product. SPAAC reactions have been established as an alternative to CuAAC and have been explored extensively as a bio-orthogonal approach for the design of various cross-linked materials.

Dey et al. have implemented SPAAC reactions using modified dPG sulfate (dPGS) as a cross-linking material to obtain biomedically relevant hydrogels with anti-inflammatory properties, beneficial for cartilage tissue engineering [38]. The anti-inflammatory activity stems from dPGS binding to L-selectin on leukocytes and P-selectin on inflamed vascular endothelium, which decreases leukocyte extravasation by shielding the adhesion molecules [39]. 


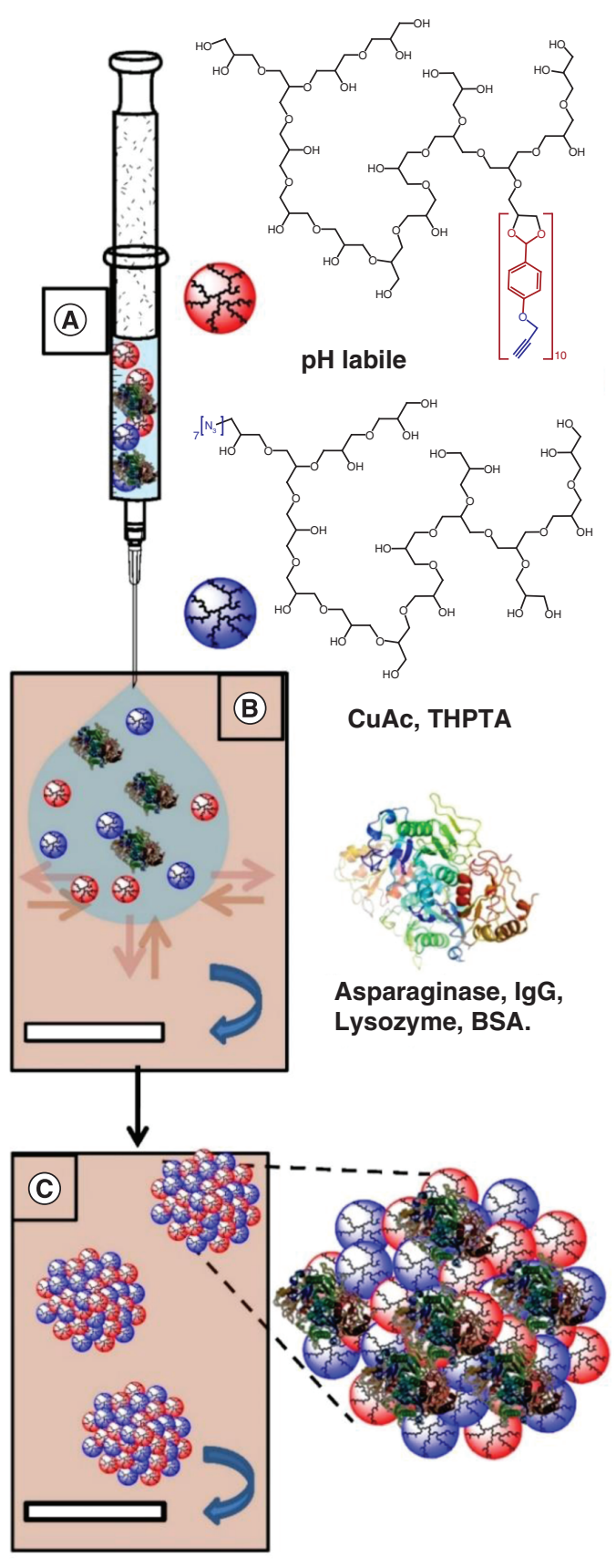

Figure 4. Use of copper-driven click chemistry and nanoprecipitation process for nanogel preparation. (A) Injection of aqueous solution of alkyne and azide functionalized polyglycerol macromonomers and the proteins asparaginase, lysozyme and bovine serum albumin. (B) Particle templation by diffusion of the aqueous phase (blue) into acetone phase (violet). (C) Nanogel formation by azide-alkyne cycloaddition.

Reproduced with permission from [36] (c) Elsevier (2013).

In addition, dPGS is well suited as a carrier for various bioactive species and is particularly interesting for tissue engineering of the cartilage due to its resemblance to heparan sulfate, one of the glycosaminoglycans found in the connective tissue of the joints.

The potential of dPGS-based hydrogels was evaluated in comparison with dPG and PEG-based hydrogels. Hydrogels containing varying amounts of dPGS were synthesized by in situ cross-linking via a SPAAC reaction using different amounts of PEG-dicyclooctyne (PEG-DIC), dPGS-N 3 and a tetra-arm star PEG-azide as macromonomers, while keeping the total polymer content constant for each hydrogel (Figure 5).

The mass swelling ratio was found to increase with the increase in the percentage dPGS content in the gels, and the viability study using encapsulated chondrocytes showed that dPGS-containing gels are more suitable for cell encapsulation than the commonly used PEG and alginate hydrogels [40]. 

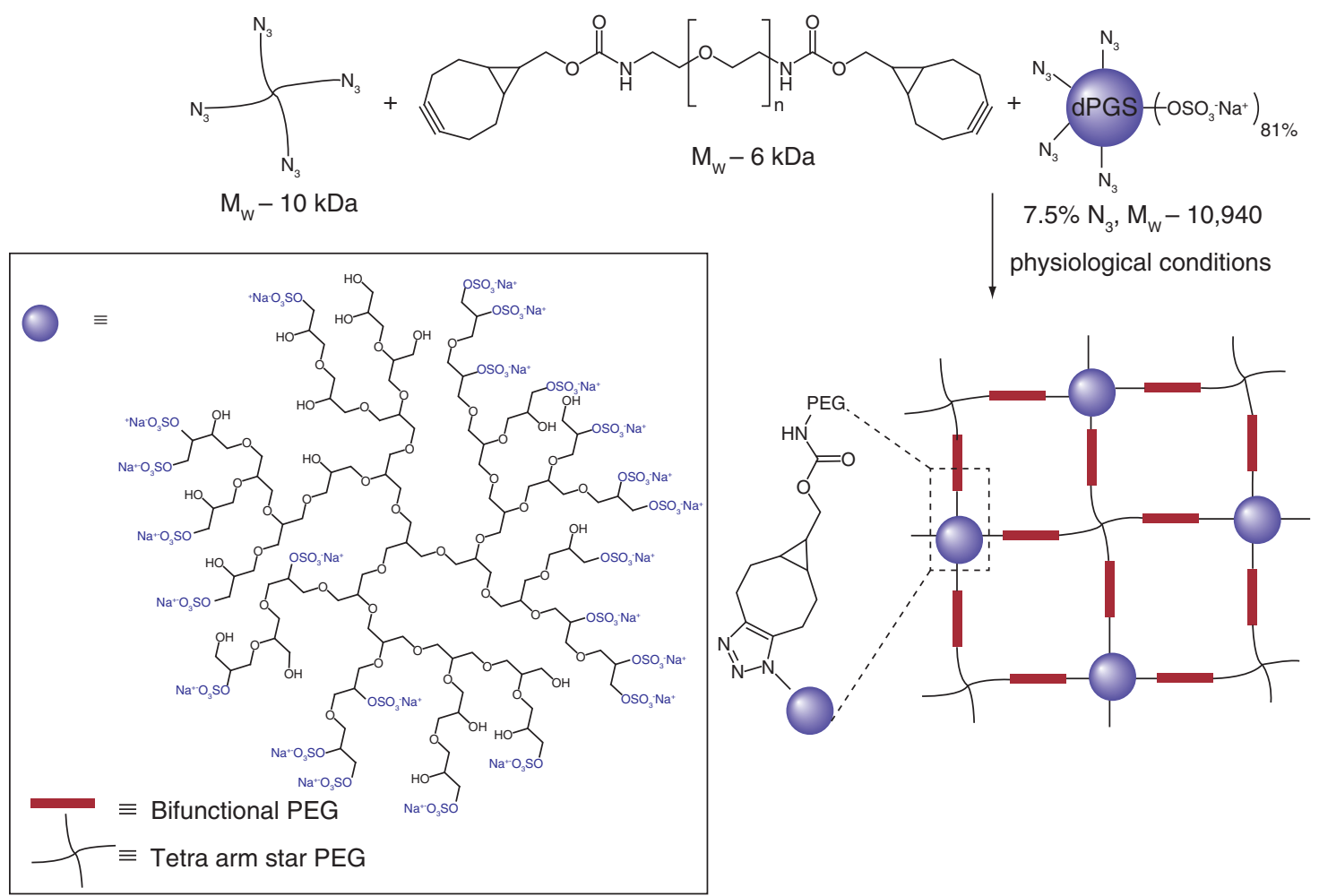

Figure 5. Structure of macromonomers used in strain-promoted azide-alkyne cycloaddition cross-linking. dPGS: Didendric polyglycerol sulfate.

Reproduced with permission from [38] (c) Wiley-VCH Verlag GmbH \& Co. KGaA (2016).

Schlaich and co-workers used the above dPGS-based hydrogel, alongside commercially available gels composed of alginate, gelatin and 4-arm PEG-vinylsulfone (4-arm PEG-VS) cross-linked with PEG-dithiol, for hydrogel bead formation on superamphiphobic and slippery surfaces [41]. The shape-controllable model system was reported for the preparation of hydrogel beads by utilizing the extreme hydrophobicity and interfacial tension of the liquid, which forces the droplets into spherical and semispherical shapes when applied on the superamphiphobic and slippery liquid-infused porous surfaces, respectively. In addition, the study demonstrated that the encapsulation of the cells into gel on the superamphiphobic surface had no impact on the viability and behavior of the cells.

To explore the impact of hydrolytic degradability on the morphology and biocompatibility of the hydrogels, a synthetic approach mediated by SPAAC reaction was designed for in situ cross-linking of dPGS- $\mathrm{N}_{3}$, star tetraarm PEG-N 3 , PEG-DIC and PEG-PCL-DIC (dicyclooctyne terminated polyethylene glycol polycaprolactone) macromonomers (Figure 6) [42]. The degradable ester linkages were introduced to allow degradation mediated by matrix metalloproteinases, an important group of protein-degrading enzymes present within the extracellular matrix [43-45]. Out of three explored hydrogels, only two contained the ester linker and out of these two, only one contained dPGS units.

All the gels were explored for in vitro degradation, and it was observed that the hydrogels containing degradable linker completely degraded under physiological conditions after 17 (dPGS-containing) and 7 (PEG units instead of dPGS) days, while the gel without this linker remained intact. The degradation behavior for PEG-based hydrogels was in line with that previously reported in the literature [46]. Cytocompatibility evaluation using mouse fibroblast L929 cells showed that in the presence of degradable hydrogels with and without dPGS, the viability of the cells was 100 and $92 \%$, respectively.

A study by Lospichl et al. presented a detailed rheological study of dPGS-based hydrogels, which were shown to be particularly well suited for intra-articular injections for the treatment of osteoarthritis [47]. The hydrogel used in the study was prepared by cross-linking PEG-dicyclooctyne (PEG-DIC) with dPGS-N 3 under physiological conditions. 


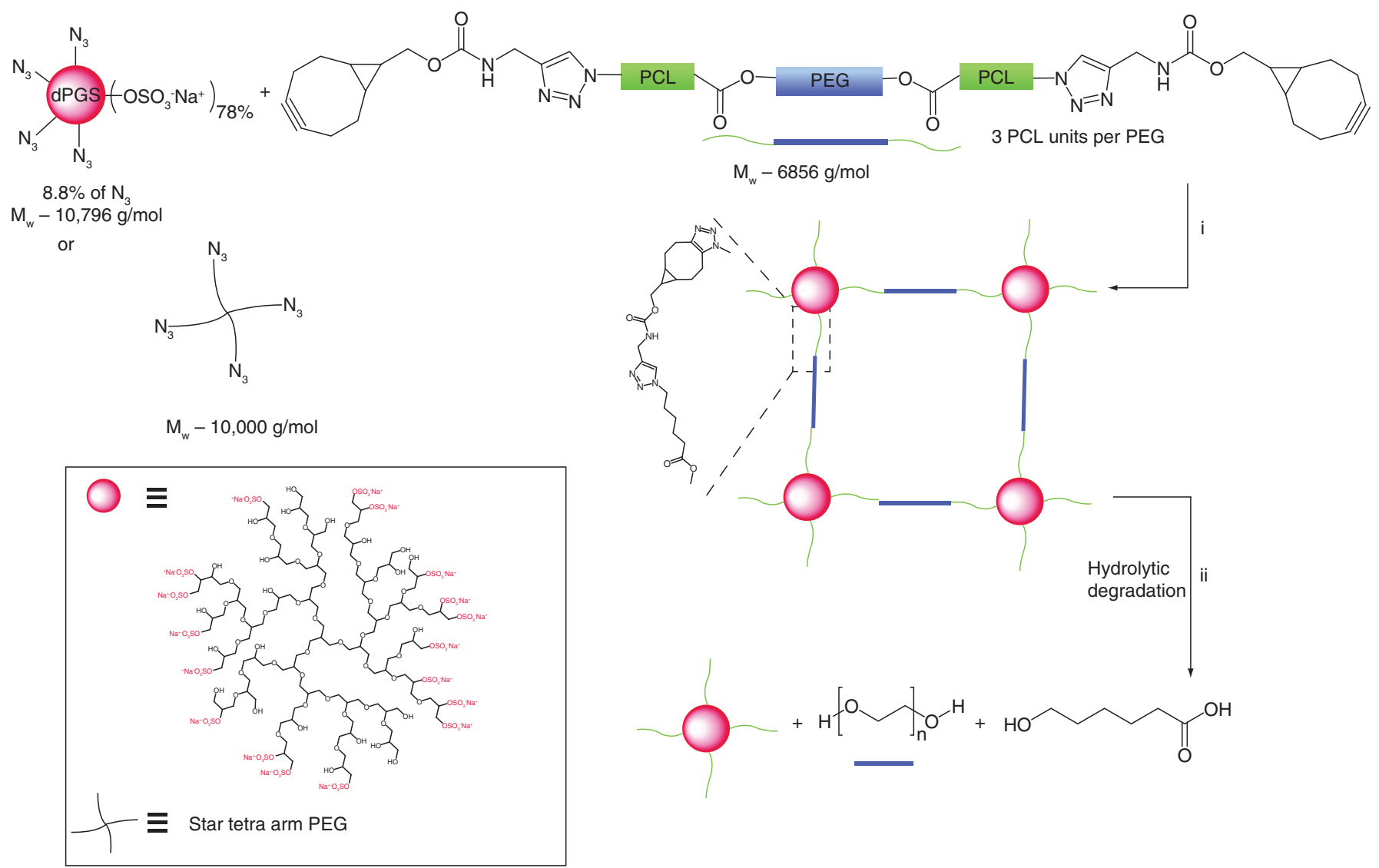

Figure 6. Formation of the covalent hydrogel networks using strain-promoted azide-alkyne cycloaddition reactions. The monomers contained an ester bond which is a substrate for matrix metalloproteinase, affording degradable hydrogels. dPGS: Didendric polyglycerol sulfate.

Reproduced with permission from [42] ๔ The Royal Society of Chemistry (2016).

In another study, Hemmati-Sadeghi and coworkers prepared a hydrogel by cross-linking of dPGS and PEGDIC, and explored the inhibition of proteoglycan loss and the expression pattern induced by recombinant porcine TNF- $\alpha$ in an in vitro model of osteoarthritis [48]. The approach involved a comparative study of both normal and osteoarthritis-induced micromasses treated with dPGS hydrogel with the help of safranin-O staining. The downregulation of TNF- $\alpha$ signaling pathways, which led to reduced depletion of glycosamionoglycans in the hydrogel-treated micromass compared with a control, provided valuable insights into the potential of dPGScontaining hydrogels for intra-articular treatment of osteoarthritis. All of these studies highlighted the potential of dPGS-containing hydrogels as functional scaffolds for cartilage tissue engineering.

Herrmann et al. used the same approach to develop biosensing materials with high sensitivity by utilizing in situ bio-orthogonal hydrogel formation via SPAAC reaction (Figure 7) [49]. The cross-linking of dPG-azide and PEGDIC yielded hydrogel networks anchored on the azide-silane functionalized surface. PEG chains of two different lengths $(3$ and $6 \mathrm{kDa}$ ) were employed to regulate the mesh size as well as the degree of flexibility of the cross-linked structures. The solution-like environment of the in situ hydrogel-based biosensor matrix enabled the encapsulation of large streptavidin protein and a smaller 20-mer oligonucleotide through noncovalent and covalent interactions. Streptavidin can be used for affinity interactions with biotinylated molecules, while encapsulated oligonucleotides are used as hybridization sensors for complementary DNA strands.

Furthermore, they described the immobilization and spatial separation of fluorescence-labelled microbeads for a multiplex detection in k-levels by utilizing a dPG-based hydrogel [50]. First, hydrogels were prepared by cross-linking dPG-N 3 with bifunctional PEG-DIC ( 6 and $10 \mathrm{kDa})$ via SPAAC reaction in the presence of microbeads. The ability of these hydrogels to act as a size-selective filter was studied using diffusion of a medium-sized fluorescently-labeled Fab fragment $(50 \mathrm{kDa})$ and large IgG-type antibody $(150 \mathrm{kDa})$ as well as two quencher-labeled oligonucleotides 


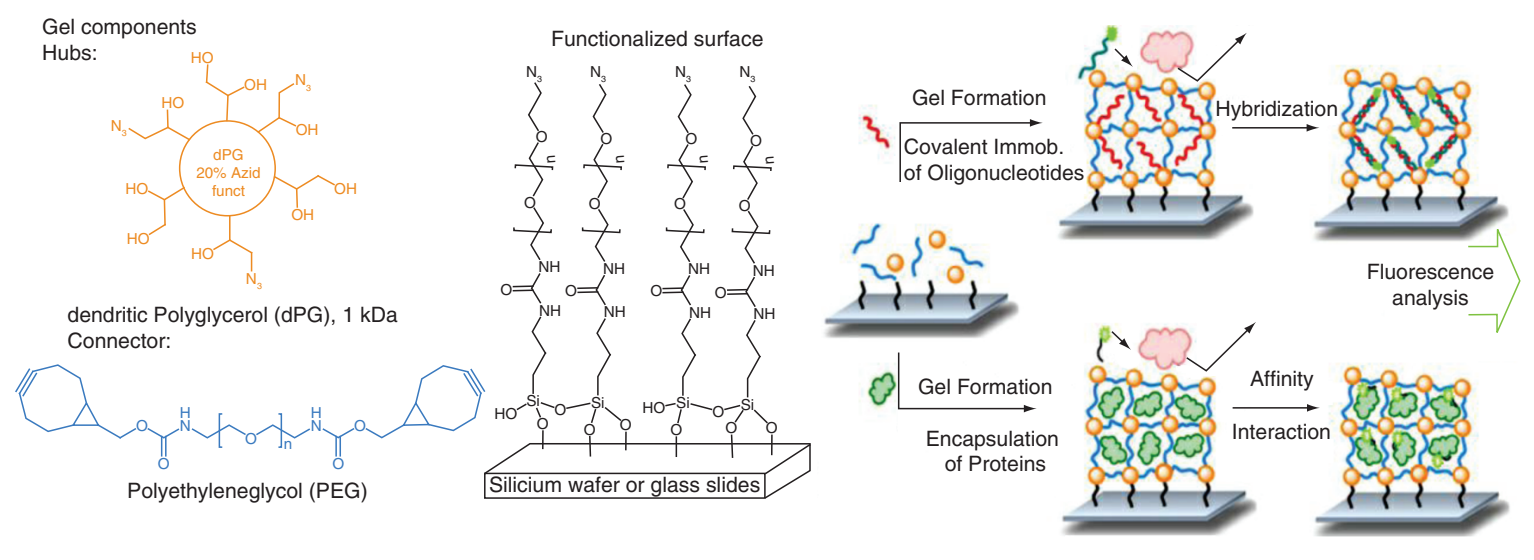

Figure 7. Gel components and hydrogel formation on glass slides using strain-promoted azide-alkyne cycloaddition reaction. Prepared hydrogels are used to embed sensing species such as protein streptavidin and single-stranded DNA to undergo affinity interaction with biotin and hybridization with complementary oligonucleotides respectively. Modified with permission from [49] (c) American Chemical Society (2018).

(19-mer, $6 \mathrm{kDa}$ and 150-mer, $46 \mathrm{kDa}$ ). While proteins represent rigid structure, oligonucleotides are flexible; 3DCoding for Compound Analysis in k Elevations (CAkE) showed that small, flexible biomolecules such as DNA diffused faster than large, rigid proteins.

In another study by Randriantsilefisoa et al., SPAAC reaction was successfully used to prepare hydrogel-based arrays in which peptides were immobilized for antibody detection. The peptide sequences used to capture the target antibody were immobilized on the azide-functionalized PEG units $(6,10,20$ and $40 \mathrm{kDa})$ to tailor the mesh size of the network, and cross-linked with cyclooctyne-functionalized dPG (Figure 8) [51]. The peptides were immobilized within the network through either thiol or amine groups and oriented in such a way as to enable antibody binding. The cross-linked hydrogel network showed not only a remarkable swelling ratio (up to 100) with up to eightfold expansion in height but also excellent elasticity, with $\mathrm{G}_{0}=200 \mathrm{~Pa}$ for a PEG $40 \mathrm{kDa}$-based gel, resulting in the softest among the reported hydrogels [52]. The higher loading capacity of the 3D hydrogel-based peptide arrays meant $20 \%$ higher sensitivity compared with 2D-based, commercially available detection assays (e.g. ELISA). In addition, the limit of detection of the 3D hydrogel-based peptide arrays was found comparable to that of ELISA.

In another report, the same authors highlighted the potential of RGD peptides (having the tripeptide sequence, Arg-Gly-Asp) anchored to soft nanocomposite hydrogels to mimic the native cell niche for maintaining the stemness and adipogenic differentiation potential of human mesenchymal stem cells (hMSCs) [53]. Different spacing between RGD units, and hydrogel stiffness - which affect the stemness and adipocyte differentiation potential of hMSCs - were achieved through the use of modified PEG units ( 6 and $20 \mathrm{kDa})$. The hydrogel was formed by cross-linking of cyclooctyne functionalized dPG with azide-terminated PEG units. The PEG and RGD groups were further anchored onto gold nanoparticles (AuNP) via thiol groups, resulting in a nanocomposite hydrogel (Figure 9). Reported studies suggested that the movement and spreading behavior of hMSCs were influenced by both the bulk and the thin film nature of the hydrogel as well as the length of the linkers. The adipogenic differentiation potential was explored on tissue culture plate polystyrene surfaces and also compared with PEG-based nanocomposite hydrogels of different softnesses. It was observed that the differentiation level increased from 20 to $80 \%$ with an increase in the softness of the surface. The interaction with hMSCs was enhanced by the larger spacing between RGD units in soft PEG-based hydrogels, which also led to a higher adipocyte differentiation potential [54].

A similar approach was further extended to design a nanocomposite hydrogel skeleton for capturing influenza $\mathrm{A}$ virus (IAV), a process which is accompanied by hydrogel shrinkage and color change [55]. This was achieved by the use of sialic acid anchored onto AuNPs, which were further linked to the PEG using Au-S chemistry (Figure 9). Sialic acid has an affinity to bind to hemagglutinin moieties on the coat of IAV through multivalent interactions [56]. The specificity for virus capturing was further enhanced by the design of the macroporous network through molecular imprinting, using the virus as a template. The dual (optical and mechanical) detection of virus binding onto the AuNPs was achieved by making use of the plasmonic properties of AuNPs [57] and the shrinking/swelling capacity of the hydrogels in the presence of the viral target. 


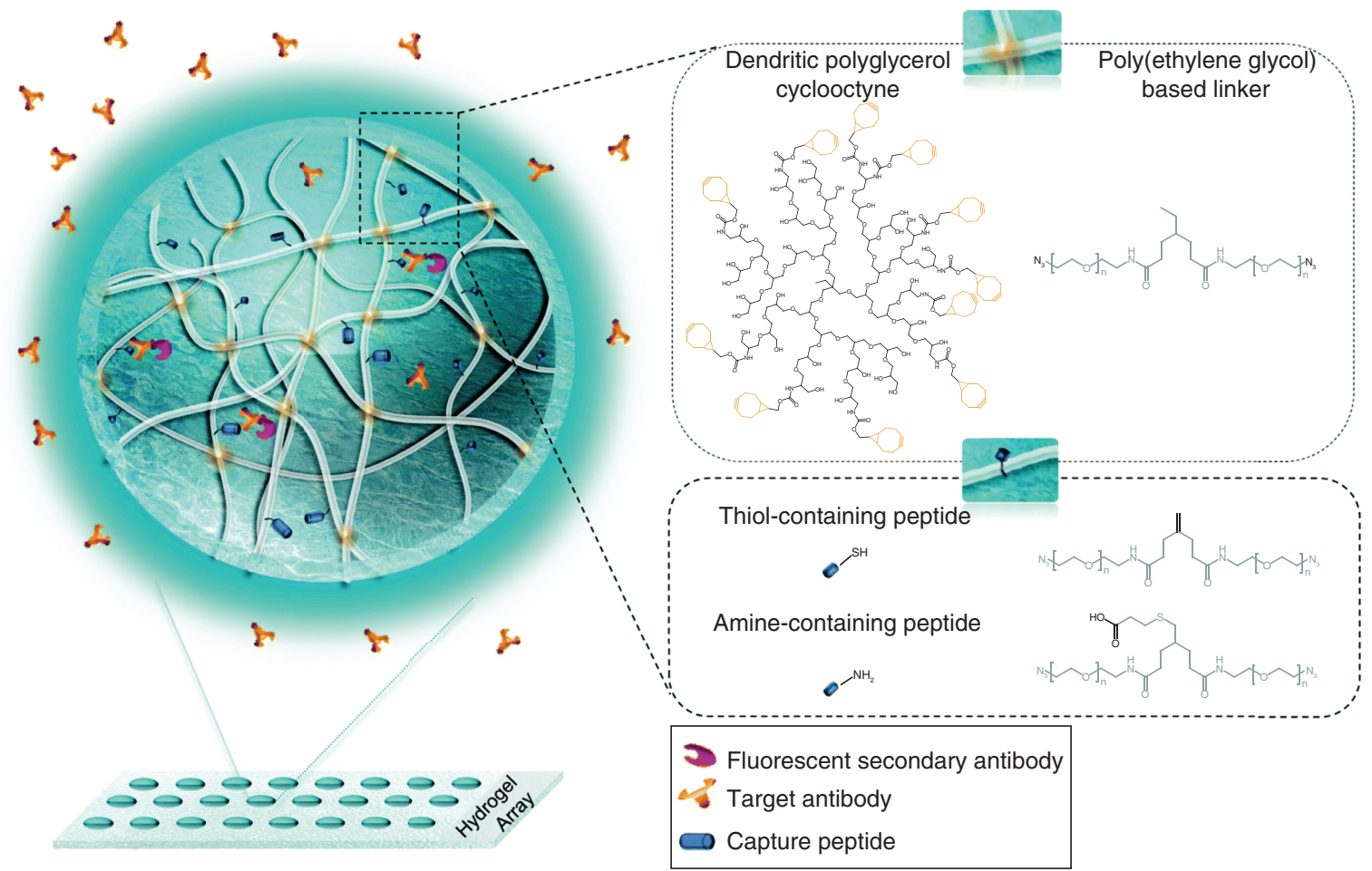

Figure 8. Hydrogel array composed of spots containing polyethylene glycol and dendritic polyglycerol-based hydrogel prepared using strain-promoted azide-alkyne cycloaddition click reaction. Polyethylene glycol units contain peptides specific to the target antibody. Hydrogel arrays are covalently attached on glass slides via silane moieties on the glass surface.

Reproduced with permission from [51] ๔ The Royal Society of Chemistry (2019).

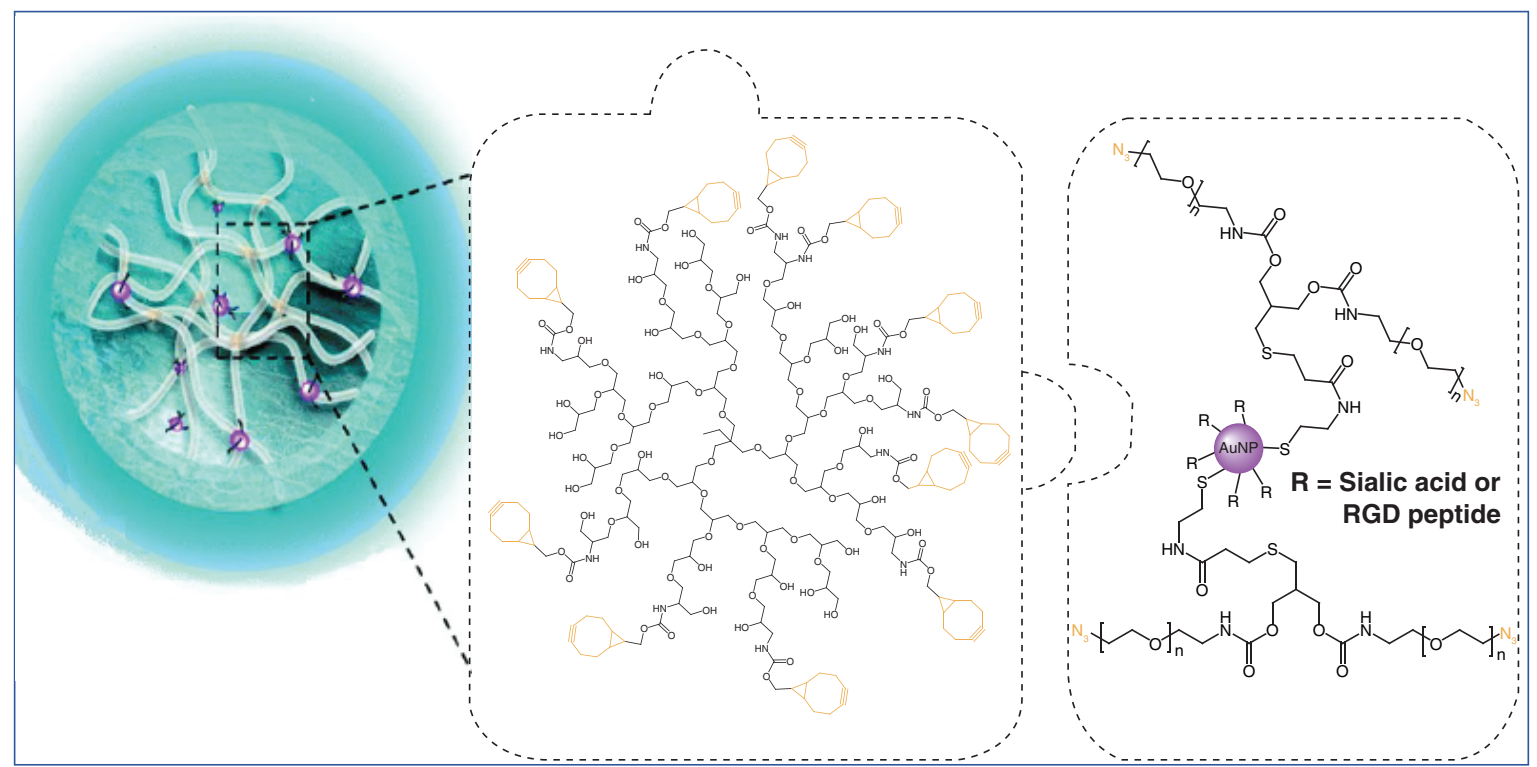

Figure 9. Hydrogel network composed of click chemistry cross-linked polyethylene glycol and dendritic polyglycerol with embedded RGD/sialic acid-gold nanoparticle conjugates.

Modified with permission from [55] (c) The Royal Society of Chemistry (2020). 


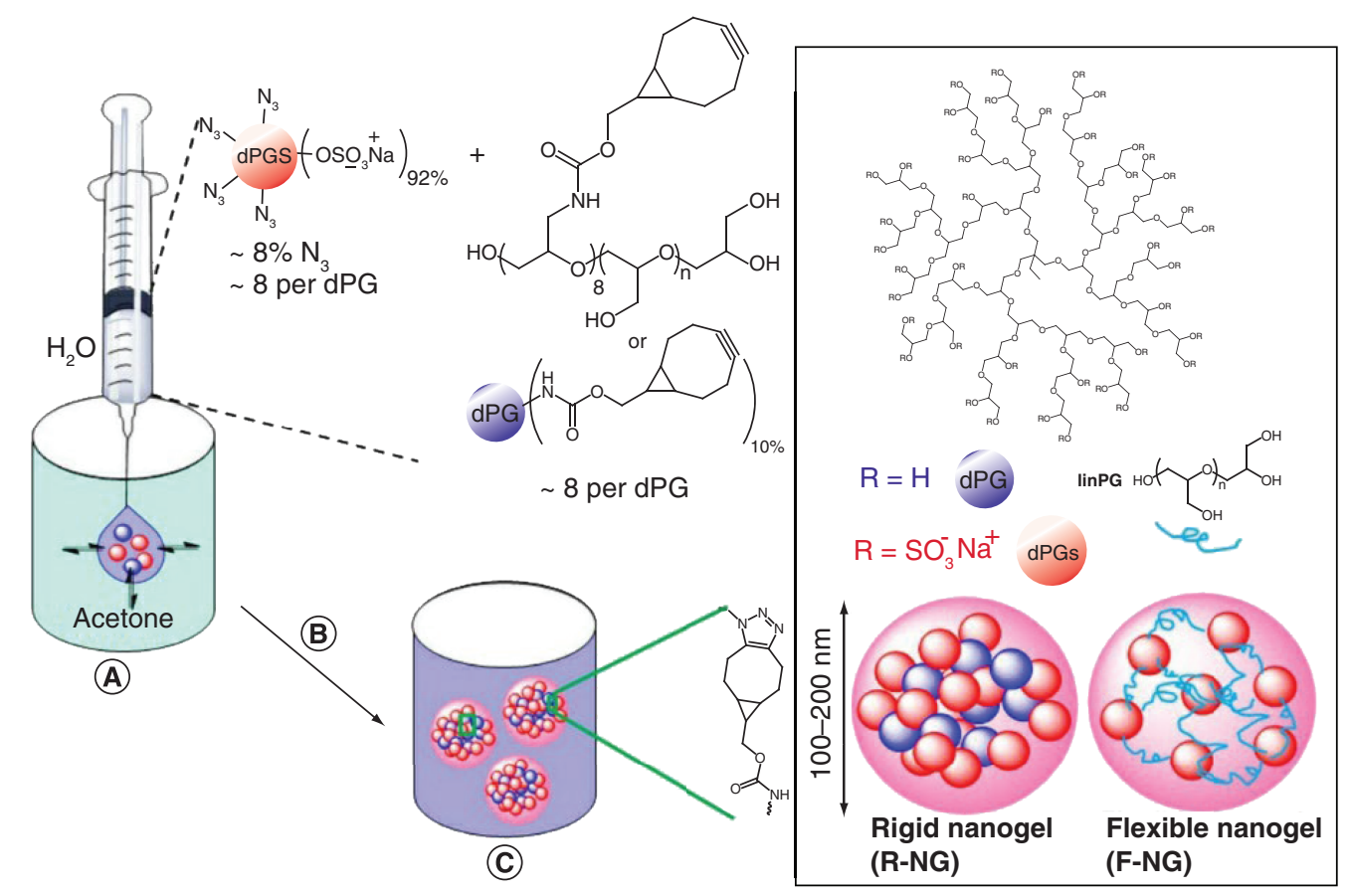

Figure 10. Rigid and flexible polyglycerol-based nanogels synthesized through strain-promoted azide-alkyne cycloaddition via inverse nanoprecipitation method, and used for capture and inactivation of herpes simplex virus. dPGS: Didendric polyglycerol sulfate.

Reproduced with permission from [58] ๔ American Chemical Society (2019).

In another virus-related work, Dey et al. designed nontoxic broad-spectrum sulfated nanogels with different degrees of flexibility based on dPGS for deactivation of herpes simplex virus [58]. Macromonomers dPGS-N $\mathrm{N}_{3}$, dPGcyclooctyne (dPG-Oct) and IPG-cyclooctyne (IPG-Oct) were utilized for cross-linking with the help of SPAAC, resulting in rigid R-nanogels (dPGS-dPG) and flexible F-nanogels (dPGS-LPG) ranging from 100 to $200 \mathrm{~nm}$ via inverse nanoprecipitation method (Figure 10). The resulting nanogels with the matching pore sizes with the sizes of different viruses and various active surface moieties, not only allowed the efficient capturing of the virus, but also enabled multivalent interactions with viral glycoproteins, leading to virus surface shielding and, ultimately, deactivation of the herpes simplex virus.

A comparative study between rigid and flexible nanogels containing sialic acid moieties in terms of the IAV inhibition was also performed [59]. It was demonstrated that the combination of flexible scaffolds and the presence of multiple virus-specific ligands on the scaffolds resulted in an adaptable 3D network which almost completely (98\%) blocked the virus adhesion onto the cells and inhibited the infection at low pM concentrations in vitro.

With the growing need for antiviral materials and structures, it could be expected that there will be an increase in the development of PG nanogel formulations.

\section{Thiol-ene \& thiol-disulfide cross-linking}

Like the SPAAC reaction, the thiol-ene reaction belongs to the family of bio-orthogonal, catalyst-free click reactions; the term refers to the reaction of the thiol group with an unsaturated double or triple bond resulting in a sulfur-carbon bond.

Employing thiol-ene cross-linking, Zabihi et al. prepared nanogel-peptide conjugates that enabled specific encapsulation of temoporfin (m-THPC), a photosensitizer used in photodynamic therapy of certain cancers [60]. The temoporfin-binding peptide was first conjugated to PG through amide coupling between its terminal carboxylic acid and amine-containing hPG (hPG-NH $\mathrm{NH}_{2}$ ) to yield nanogel precursor hPG-Pep (Figure 11). Subsequently, amino functional groups of hPG-Pep were converted to thiols using 2-iminothiolane hydrochloride (Traut's reagent) in order to activate hPG-Pep for the thiol-click reaction. In an attempt to investigate the effects of peptides on interactions between nanogels and m-THPC, for each nanogel-peptide conjugate, its analogue without peptides was synthesized using thiol-ene click reaction and studied for their interactions with m-THPC. It was observed that 


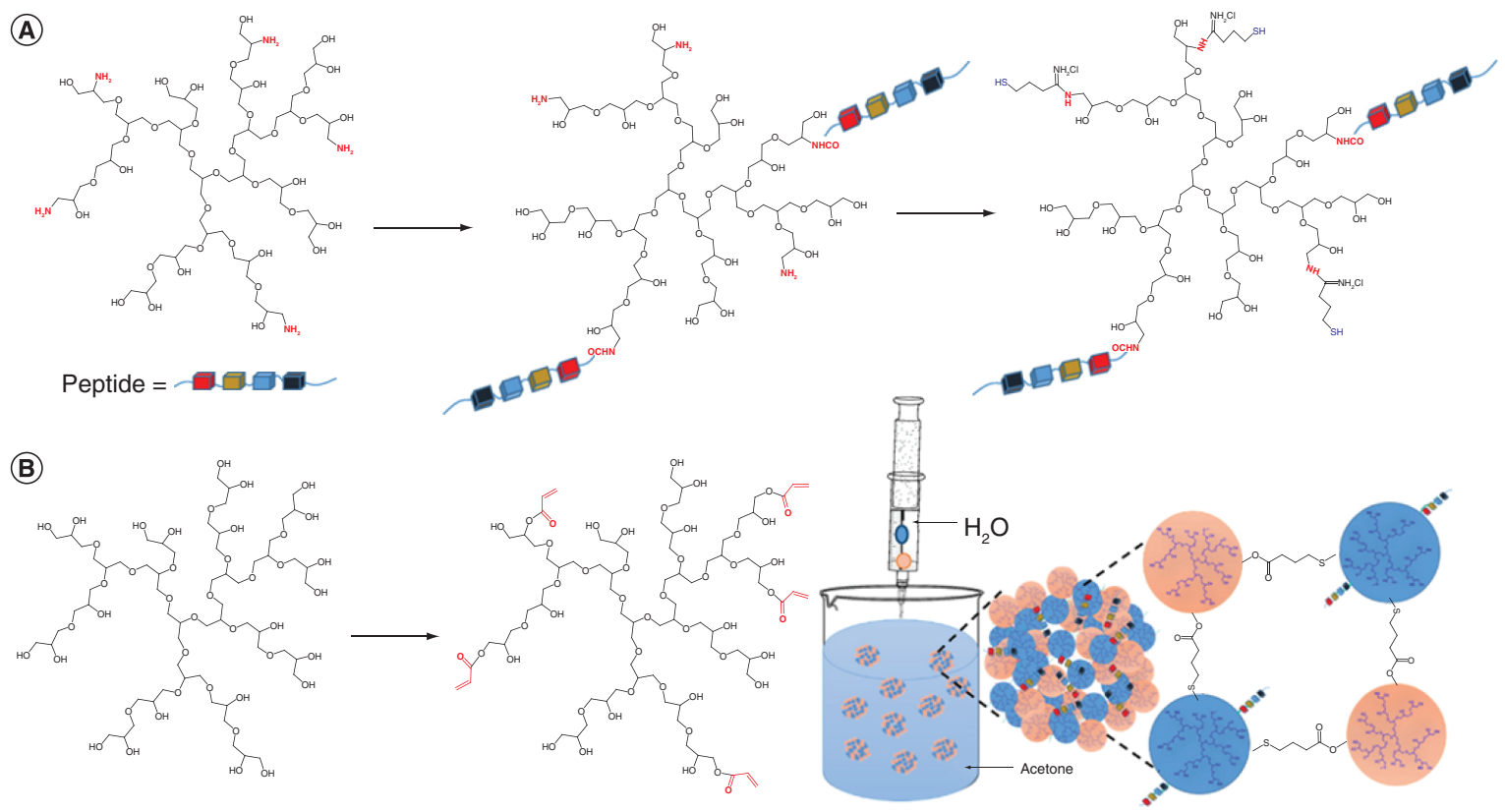

Figure 11. Synthesis of peptide-conjugated thiol-ene cross-linked nanogel. (A) Functionalization of amine-containing polyglycerol with peptide and subsequently with thiol moiety. (B) Acrylation of polyglycerol. (C) Nanoprecipitation of thio-peptide-functionalized polyglycerol and acryl-functionalized polyglycerol. Modified with permission from [60] (c) Elsevier (2014).

upon peptide conjugation, the loading capacity of nanogels improved 16-times. Also, nanogel-peptide conjugates enhanced the penetration of $\mathrm{m}$-THPC in viable skin layers, as evidenced by skin penetrations tests in barrierdeficient skin.

In another application, $\mathrm{pH}$-sensitive nanogels in the $<100$-nm range were designed using thiol-ene cross-linking and applied to gene delivery [61]. $\mathrm{pH}$ sensitivity was achieved using the $\mathrm{pH}$-sensitive $\mathrm{dPG}$-amine, which was first thiolated (resulting in thiol-dPG), then coupled with polyethylenimine-acrylamide (PEI-acrylamide) via a thiol-ene nanoprecipitation process under mild conditions and in the absence of any catalyst or surfactant (Figure 12A). The biocompatible conditions of this inverse nanoprecipitation process allowed for in situ encapsulation of sensitive siRNA. Furthermore, the properties of siRNA encapsulated within the nanogel were compared with those of siRNA complexed with polyplex nanogel (NGp) shortly before usage. It was observed that the amalgamation of $\mathrm{pH}$-sensitive benzacetal bonds inside the nanogel network enabled the controlled intracellular release of the cargo, as demonstrated by siRNA-mediated silencing of green fluorescent protein (GFP) expression in HeLa cells (up to $71 \%$ silencing was achieved). This indicated that nanogels could be a suitable platform not only for gene therapy but also possibly for DNA vaccine design.

The authors further developed larger $\mathrm{pH}$-responsive dPG-NGs $(0.1-1 \mu \mathrm{m})$ with a narrow polydispersity to determine the $\mathrm{pH}$ values inside the hair follicle using an ex vivo porcine ear model [62]. The macromolecular precursor dPG-amine was conjugated with a $\mathrm{pH}$-sensitive indodicarbocyanine dye and a control indocarbocyanine dye through N-hydroxysuccinimide ester coupling, with subsequent in situ thiolation and coupling with dPGacrylate (Figure 12B). The cross-linking occurred via a pseudo-click thiol-Michael reaction using the inverse nanoprecipitation method. Automated analysis of confocal microscope images of histological sections of the skin enabled precise determination of the $\mathrm{pH}$ gradient inside the follicle; the results revealed that the nanogel sensor could penetrate the skin through the follicular pathway and showed no toxicity. In addition, it could be used to determine the $\mathrm{pH}$ gradient in the hair follicles, which ranged from 6.5 at the surface of the skin to 7.4 in deeper follicle layers.

The formation of protein coronas on dPG-based nanogels and their impact on cellular uptake, drug release, toxicity and immunogenicity were studied by Obst et al., wherein corona-bearing nanogels induced marked escalation in cytokine release from primary human macrophages with no apparent enhancement in in vitro toxicity [63]. 
(A)

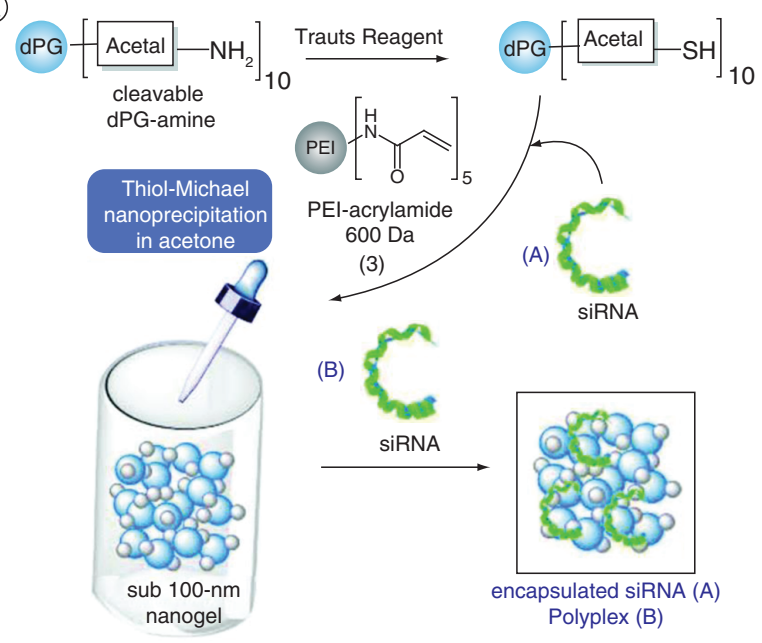

(B)

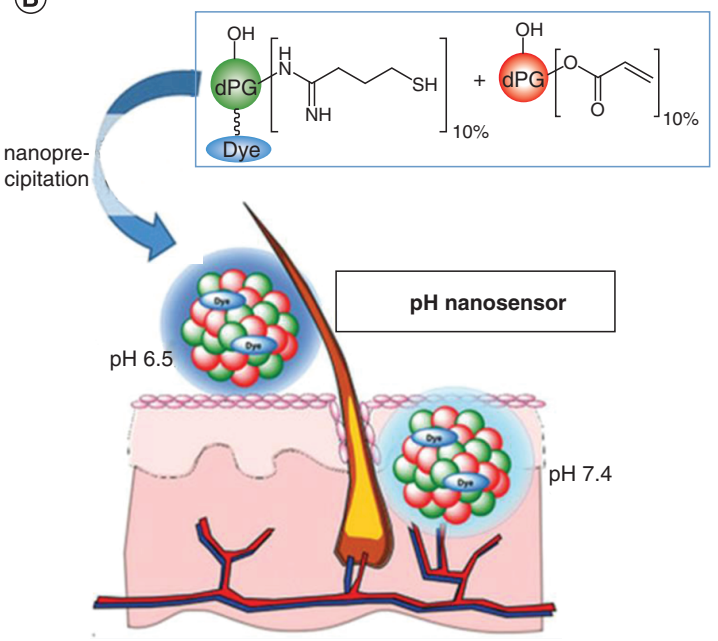

Figure 12. Synthesis of thiol-ene cross-linked nanogels. (A) Design of a pH-responsive polyglycerol-PEI nanogel using thiol-ene cross-linking and nanoprecipitation in acetone. The resulting nanogels can be used to encapsulate siRNA and induce green fluorescent protein silencing upon release within HeLa cells. (B) pH-sensitive nanogel used as $\mathrm{pH}$ nanosensor for skin and hair follicle prepared by thiol-ene cross-linking of two monomeric elements. Dye embedded within the gel acts as a fluorescent $\mathrm{pH}$ sensor, ensuring precise measurement of $\mathrm{pH}$ values at different layers of skin and follicle.

PEl: Polyethylenimine.

(A) Reproduced with permission from [61] (c) The Royal Society of Chemistry (2017). (B) Reproduced with permission from [62] () Wiley-VCH Verlag GmbH \& Co. KGaA (2017).

Inverse nanoprecipitation was also employed by Zhang et al. to obtain a hydrophilic prodrug nanogel responsive to $\mathrm{pH}$ and redox changes [64]. This was achieved by the addition of dithiothreitol to the synthesized PG-thioctic acid, which cleaved the lipoyl rings, initiating a thiol-disulfide exchange reaction and the formation of disulfide cross-linked networks (Figure 13). Intracellularly degradable $\mathrm{pH}$ - and redox-sensitive nanogels carrying the chemotherapeutic agent doxorubicin $(\mathrm{DOX})-\mathrm{NG}-\mathrm{DOX}_{\mathrm{i}}$ with DOX in the interior and NG-DOX with DOX on the surface of the nanogel - were then prepared. NG-DOXi was designed using PG conjugated to doxorubin modified with a maleimide-containing acid-labile linker (DOXO-EMCH) through thiol-ene Michael addition. Inverse nanoprecipitation of doxorubicin-conjugated PG in the presence of dithiothreitol resulted in nanogels with

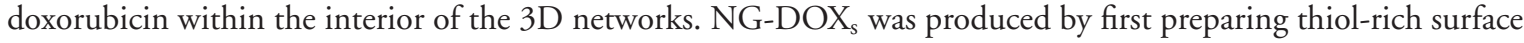
which was subsequently reacted with DOXO-EMCH. Both nanogels had a size of approximately $150 \mathrm{~nm}$ and were able to accumulate in tumor tissue through the enhanced permeation and retention effect, had a very low level of drug leaching and demonstrated efficient intracellular release of the payload triggered by the intracellular conditions.

\section{Boronic acid-diol cross-linking for hydrogel formation}

Boronic acids reversibly form boronate ester complexes with 1,2- or 1,3-diols and are well suited for crosslinking of 1,2-diol-containing PG monomers. Using this strategy, Zhang et al. developed a degradable nanogel system responsive to ATP and $\mathrm{pH}$ using a mild, surfactant-free inverse nanoprecipitation method employing PG monomers and boronic acid containing PEG-PG cross-linker (Figure 14A) [65]. Coprecipitation with the anticancer drug methotrexate (MTX) resulted in a methotrexate-loaded nanogel with a loading capacity of $13 \mathrm{wt} \%$ and sizes that ranged from 100 to $300 \mathrm{~nm}$. Rapid release of the drug was observed upon addition of ATP and in acidic $\mathrm{pH}$, as well as a dose-dependent effect on HeLa and MCF-7 cancer cells.

In another report, an ATP- and $\mathrm{pH}$-sensitive boronate ester cross-linked zwitterionic nanogel (NG-CA) was prepared and used for the delivery of therapeutic protein (Figure 14B) [66]. A dPG amine-boronic acid conjugate (dPGA-FPBA) was coupled to citraconic anhydride (CA) through amide coupling of the free amines, resulting in a CA-conjugate (PGA-FPBA-CA) that was stable under neutral or basic conditions but cleavable at acidic $\mathrm{pH}$. Using citraconic PG as a cross-linker and dPG as a scaffold monomer, zwitterionic nanogels (NG-CA) were obtained which were able to flip charges depending on $\mathrm{pH}$. Following the design of the monomers a therapeutic 


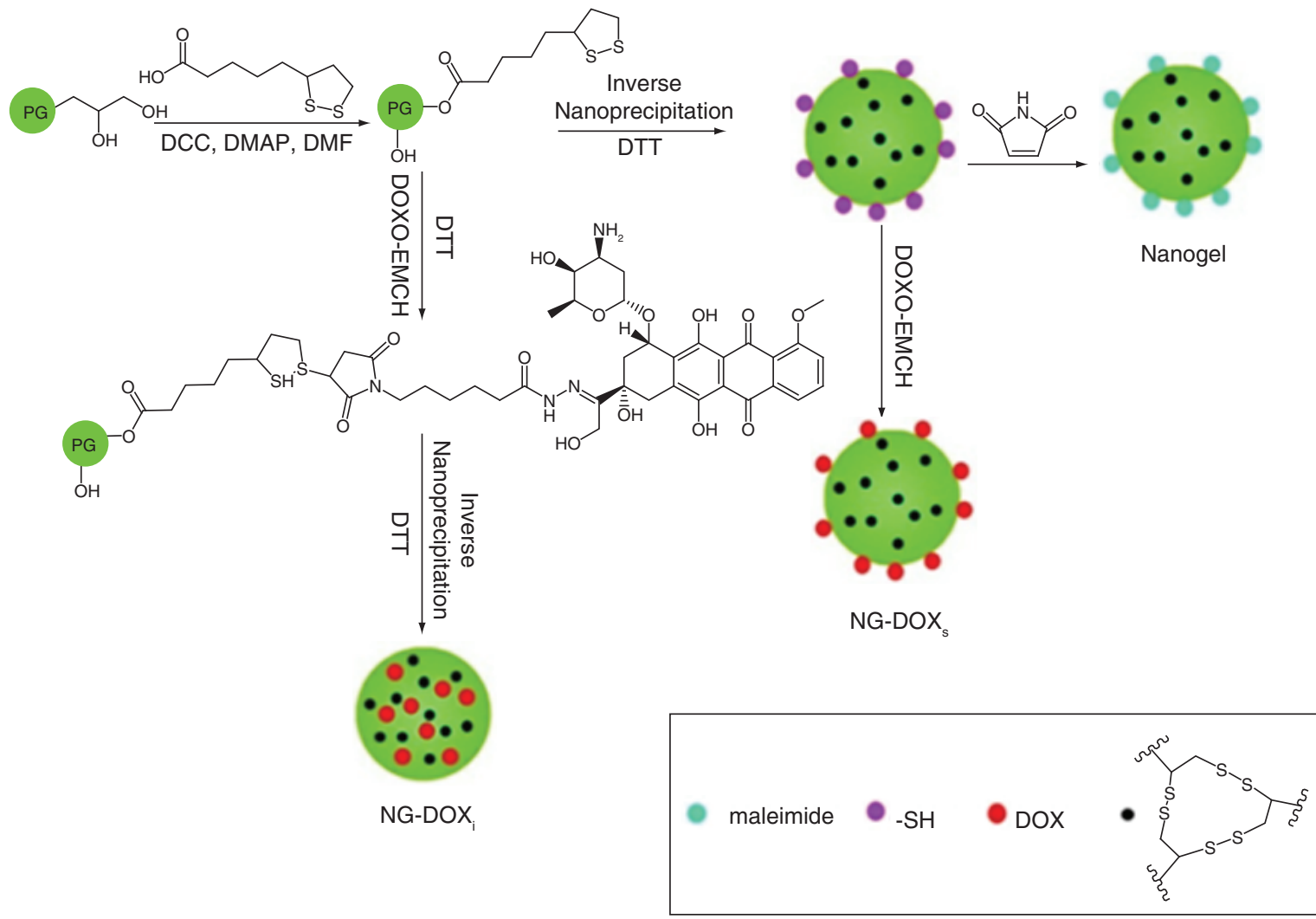

Figure 13. Nanogels depicting both the embedding and conjugation of the chemotherapeutic drug doxorubicin in the interior and to the thiol moieties on the exterior surface, respectively. DOXO-EMCH refers to modified doxorubicin containing maleimide and acid-labile linker-conjugated doxorubicin. Reproduced with permission from [64] (c) Elsevier (2014).

protein, cationic cytochrome C, was encapsulated into NG-CA via inverse nanoprecipitation through electrostatic interactions to form a protein-loaded nanogel. Once within the acidic organelles (e.g., endosomes), citraconic amide was cleaved, exposing positively charged amines that facilitated endosomal escape. The presence of ATP and acidic conditions also accelerated the release of the protein.

\section{Acid catalyst-mediated synthesis of nanogels}

Redox-labile disulfide bonds are promising biodegradable linkers that offer an approach to achieve post-endocytic nanoparticle degradation. Moreover, the fact that disulfide bonds are typically stable in the largely oxidative extracellular media enables site-specific cleavage of internalized nanoparticles and the possible release of active agents to the cytoplasm. Taking into account the benefits of disulfide bonds, Steinhilber $e t$ al. reported the synthesis of biodegradable nanogels with a controllable dimension and bearing a disulfide-linked PG structure [67]. Such PG-disulfide nanogels were prepared using acid-catalyzed inverse miniemulsion polymerization that employed a dispersion of polar reactants in nonpolar cyclohexane, various monomers (glycerol, glycerol tris-glycidyl ether and disulfide derivatives), poly(ethylene-co-butylene)-block-poly(ethylene oxide) surfactant and a small amount of DMSO (Figure 15). Using this strategy, particles ranging from 25 to $350 \mathrm{~nm}$ were obtained, which degraded into smaller oligomeric units in a reducing intracellular environment.

In another study, acid-catalyzed epoxide-opening polymerization was employed to prepare a series of polyether nanogels ranging from 40 to $120 \mathrm{~nm}$ from inexpensive and commercially available glycerol/oligoglycerol monomers with bis-/tris-epoxides as cross-linkers [68]. Using DMSO as an osmotic pressure agent in order to stabilize the emulsion against Ostwald ripening and poly(ethylene-co-butylene)-block-poly(ethylene oxide) as a surfactant, a controlled particle size of highly branched polyether polyol nanogels could be achieved. In addition, by changing the functionalities of the monomers and cross-linkers, the properties of these nanogels (e.g., size, degree of branching, viscosity and swelling behavior) could be controlled. 
(A)
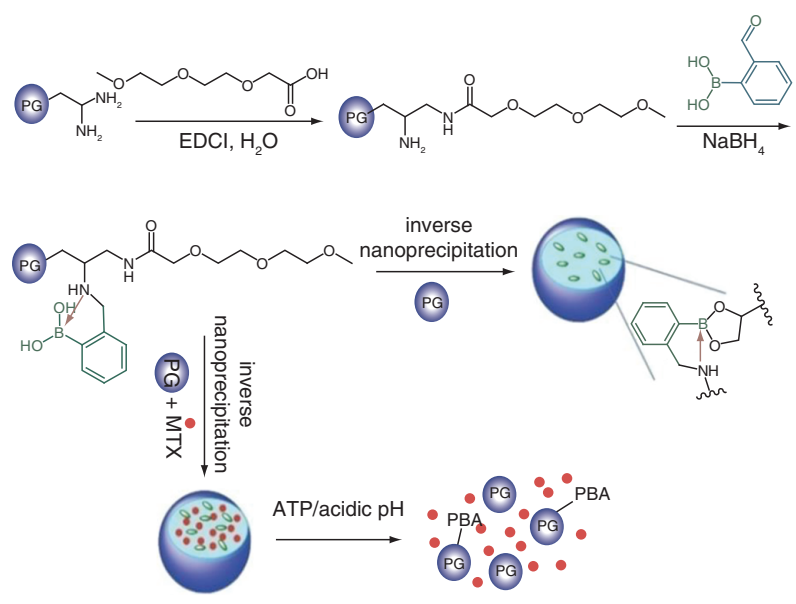

(B)
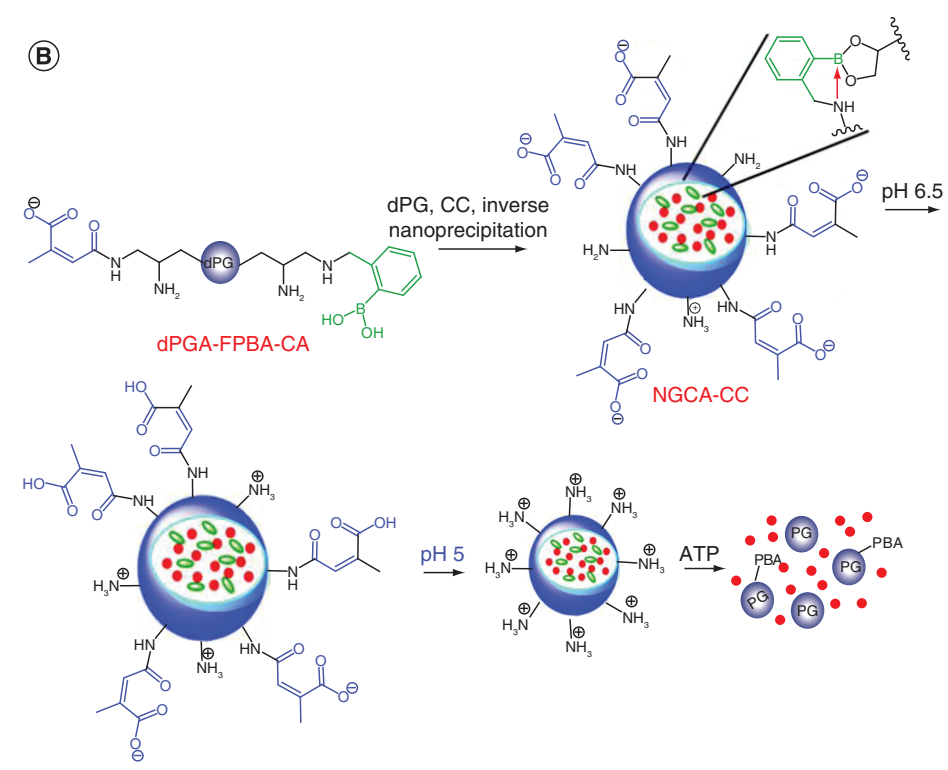

Figure 14. Synthesis of boronic acid-diol cross-linked nanogels. (A) Synthesis of drug carrier polyglycerol nanogels sensitive to ATP and acidic $\mathrm{pH}$, using boronic acid-diol interaction. Methotrexate is embedded within the nanogel using inverse nanoprecipitation and released in the presence of ATP and in the acidic $\mathrm{pH}$ conditions often encountered within cancer cells. (B) ATP and $\mathrm{pH}$-sensitive nanogel with embedded cationic cytochrome $\mathrm{C}$ protein. Negatively charged groups are cleaved at low $\mathrm{pH}$, exposing positively charged amines which facilitate endosomal escape.

dPG: Didendric polyglycerol.

(A) Modified with permission from [65] ( Wiley-VCH Verlag GmbH \& Co. KGaA (2015). (B) Modified with permission from [66] (c) The Royal Society of Chemistry (2015).

Hellmund et al. reported a series of 100-nm PG-amine nanogels with different amine densities synthesized by acid-catalyzed epoxide-opening polymerization of glycidol using a miniemulsion approach and follow-up surface modification to afford amine groups on the surface (Figure 16) [69]. High amine density corresponded to high DNA binding affinity and low cytotoxicity, and cellular uptake studies demonstrated the potential of such systems for drug and gene delivery applications.

\section{Other cross-linking methods for PG hydrogel/nanogel preparation}

Other strategies were employed for nanogel formation, although not as extensively as the methods discussed above. Recently, a supramolecular polymer nanogel was designed using pillar[5] arene and alkyl chains on an hPG backbone as cross-linking agent to afford a novel dermal drug delivery system [70]. These polymer networks showed significantly high drug loading capacity, with slower drug release and with higher skin penetration than formulations involving individual polymers or a conventional cream.

Oehrl et al. utilized IEDDA-based click chemistry for cross-linking [71]. To achieve the cross-linking, a water-stable tetrazine derivative, 4-(6-methyl-1,2,4,5-tetrazin-3-yl)benzoic acid, was conjugated to a dPG-amine core through an amide bond to form dPG-methyl tetrazine (dPG-metTet). Different dPG-dienophiles were subsequently crosslinked with dPG-metTet via inverse nanoprecipitation, leading to the synthesis of nanogels (Figure 17).

Particularly useful, although not yet employed in PG hydrogel/nanogel preparation, would be light-triggered click chemistry strategies such as nitrile imine-mediated tetrazole-ene cycloaddition [72] or photoenol strategy [73], which would enable more control over cross-linking as well as the spatial and temporal changes in mechanical properties.

\section{Conclusion}

Hydrogels and nanogels have huge potential for biomedical applications, both as drug delivery systems and as scaffolds for extracellular matrix design in tissue engineering. To meet the demands of continuous medical developments, it is important to continue improving the synthesis of hydrogels and nanogels to yield more controllable, scalable and affordable systems. In this review we have attempted to give an overview of PGbased hydrogels and nanogels, which emerged as excellent platforms for diverse biomedical applications. Because 

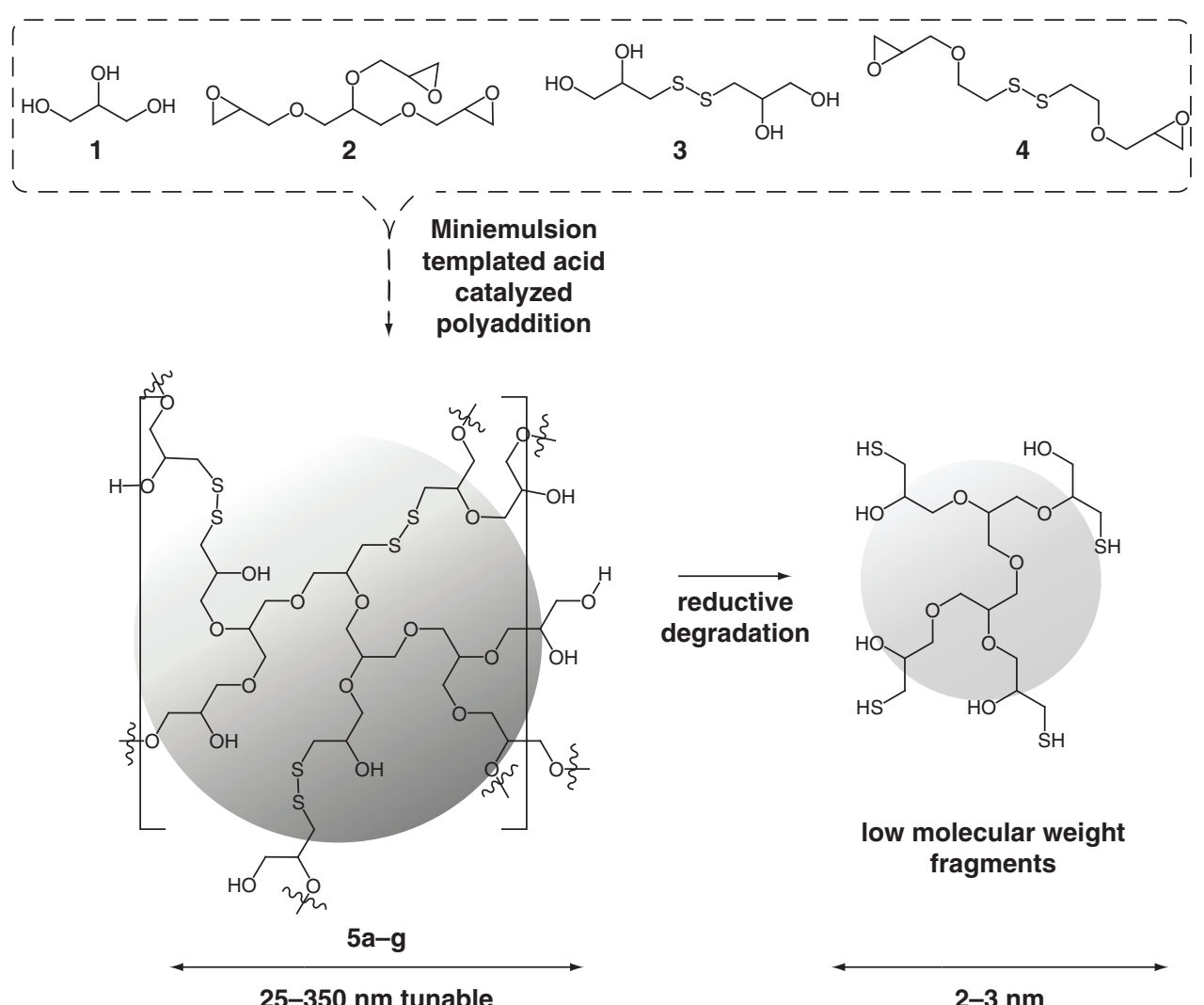
low molecular weight fragments

2-3 nm

Figure 15. Biodegradable polyglycerol nanogel containing disulfide bonds, which ease the degradation into smaller fragments in reductive environments such as within the cell.

Reproduced with permission from [67] (c) Wiley-VCH Verlag GmbH \& Co. KGaA (2010).

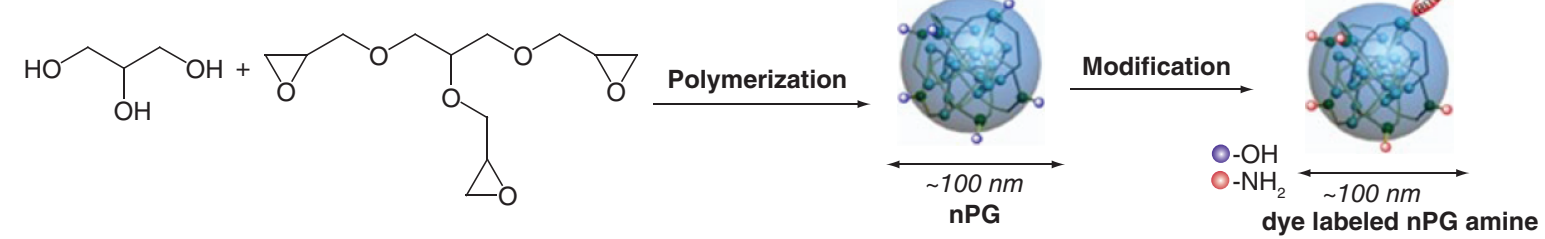

Figure 16. Acid-catalyzed epoxide-opening polymerization of glycidol and subsequent surface modification to prepare nanogels with high surface density of amine groups suitable for DNA immobilization.

Modified with permission from [69] (c) Wiley-VCH Verlag GmbH \& Co. KGaA (2010).

of their excellent biocompatibility, water-solubility and multiple functionalities, PGs can be formulated into drug carriers, and their properties tuned to enable pathogen inhibition or cell growth. Particularly well-suited systems are those based on covalently cross-linked gels, which have superior mechanical properties, sufficient stability to aid the delivery of drug cargo to the targeted sites, and tunable properties that enable controllable cargo release. With the development of tissue engineering strategies that enable the growth of multiple cell lines in three dimensions, hydrogels with multiple anchoring points enable better control and more realistic biological environments. Conventional click chemistry strategies have been extensively employed in recent years to design a variety of PG hydrogels and nanogels, but the field is far from exhausted. With the introduction of more biomolecular species such as proteins, nucleic acids and antibodies, further strategies - such as light-triggered click chemistry or protein tag strategies - will need to be introduced to afford mild coupling and ease of conjugation/encapsulation. 


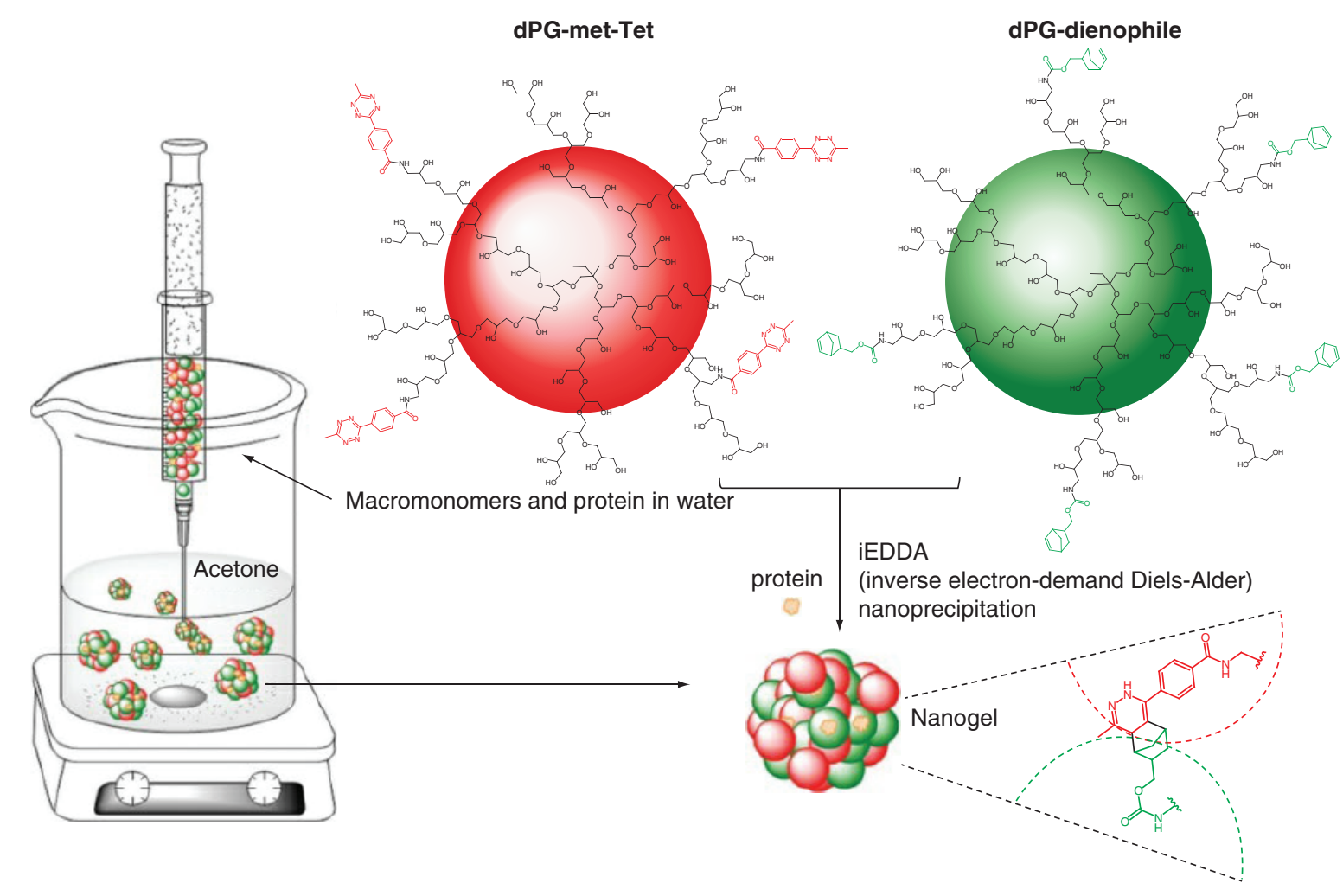

Figure 17. Nanogel formation via inverse nanoprecipitation of dendritic polyglycerol-methyl tetrazine with dendritic polyglycerol-norbonene and showing possible encapsulation of protein. Reproduced with permission from [71] @ Wiley-VCH Verlag GmbH \& Co. KGaA (2020).

\section{Future perspective}

This review of the compiled research work related to PG-based hydrogels and nanogels establishes the vast potential of dPG/dPGS-based cross-linked architectures for a wide range of applications. Although adaptable systems responsive to cell environments (e.g., the presence of enzymes) were already prepared, the use of biomolecular stimuli to control the cross-linking and the properties of hydrogels is still in its infancy. The potential in that area is vast, and future research should focus on the exploration of enzyme biomarkers characteristic for a particular tissue, and their use in synthesis and hydrogel property tuning. One particular class of enzymes that should be explored are the metalloproteinases identified in a range of cancer tissues. Their ability to cleave small peptide linkers would provide an excellent platform for the design of metalloproteinase-tunable hydrogels. Researchers working in the field of hydrogel design need to strengthen their interaction with molecular biologists to find suitable ligands and develop linkers that will improve cell proliferation and enable encapsulation of cell nutrients for healthy growth, and also to develop suitable drug release strategies. In the light of the current COVID-19 pandemic, interesting pathogen-capturing hydrogels with embedded drug cargo could be designed; the efficiency of the reported crosslinked architectures toward inhibition of IAV and herpes simplex virus has opened new routes to the exploitation of PG hydrogel/nanogel formulations in containing viral infections. In order to deal with new pathogens, we will need to design systems that can both capture and destroy, and if possible, act on-demand. This will require improved synthetic strategies and harvesting of molecular biology knowledge, particularly exploiting the activity of specific enzymes. The remarkable synthetic flexibility of the PG hydrogels - combined with new biodecoration strategies inspired by the latest developments in molecular biology, virology and cancer biology - will allow for the design of smarter, on-demand, dual-action hydrogels to be used for tissue engineering but containing both diagnostic and therapeutic units: theranostic hydrogels. Such hydrogels will largely benefit from the incorporation of new classes of nanoparticles within the hydrogel structure, which has already been shown to contribute to both the mechanical and the function-related gel properties. The future of PG hydrogels is bright, but will require joint interdisciplinary efforts. 


\section{Executive summary}

- Polyglycerol (PG) macromonomers can be used as efficient building blocks for fabricating 3D cross-linked networks (hydrogels and nanogels) owing to their hydrophilicity, excellent biocompatibility and ease of functionalization.

- The PG cross-linked architectures exhibit high swelling due to their hydrophilic nature, tunable mechanical properties (rigidity/flexibility) and the ability to encapsulate a large number of guest molecules and release them on demand using cleavable linkers.

- The most frequently used synthetic methods for the design of PG hydrogels and nanogels involve enzyme-mediated cross-linking, click chemistry approaches (copper-catalyzed azide-alkyne cycloaddition, strain-promoted azide-alkyne cycloaddition, thiol-ene and thiol-disulfide cross-linking), boronic acid-diol-based cross-linking, acid-mediated cross-linking and inverse electron demand Diels-Alder cycloaddition. New strategies are continuously being introduced.

- The enzyme-mediated cross-linking achieved with the help of horseradish peroxidase and Novozym ${ }^{\circledR} 435$ not only offers excellent selectivity, but also yields uniformly cross-linked architectures without any toxic by-products.

- Among the different click reactions, strain-promoted azide-alkyne cycloaddition has been explored extensively owing to its ability to occur under physicochemical conditions without the production of toxic by-products.

- The designed hydrogels/nanogels have been used successfully for antibody detection, encapsulation of various biomolecular cargo (proteins, peptides, oligonucleotides, genes and a range of drugs) as well as inhibitors of viral infections (herpes simplex virus and influenza A).

- Hydrogels with modified dendritic PG sulfate were successfully used in the treatment of osteoarthritis owing to their anti-inflammatory properties.

- The incorporation of $\mathrm{pH}$-labile and $\mathrm{pH}$ - and redox-sensitive moieties within linear/dendritic PG and dendritic PG sulfate enabled control of the hydrogel's biodegradability as well as site-specific cargo release.

- The incorporation of gold nanoparticles into dendritic PG/dendritic PG sulfate resulted in nanocomposite hydrogels, which were capable of capturing, detecting and removing influenza A virus. The use of virus-specific ligands such as sialic acid enhanced the antiviral potential of the hydrogel, which was achieved through the blocking of virus adhesion onto cells.

- The overview of various synthetic techniques and applications clearly indicates the huge potential of PG-based hydrogels and nanogels for use in the design of theranostic hydrogels and multifunctional tissue engineering systems.

Financial \& competing interests disclosure

The authors have no relevant affiliations or financial involvement with any organization or entity with a financial interest in or financial conflict with the subject matter or materials discussed in the manuscript. This includes employment, consultancies, honoraria, stock ownership or options, expert testimony, grants or patents received or pending, or royalties.

No writing assistance was utilized in the production of this manuscript.

\section{References}

Papers of special note have been highlighted as: $\bullet$ of interest

1 El-Sherbiny IM, Yacoub MH. Hydrogel scaffolds for tissue engineering: progress and challenges. Glob. Cardiol. Sci. Pract. 2013(3), 316-342 (2013).

2 Caló E, Khutoryanskiy VV. Biomedical applications of hydrogels: a review of patents and commercial products. Eur. Polym. J. 65, 252-267 (2015).

3 Wichterle O, Lím D. Hydrophilic gels for biological use. Nature 185(4706), 117-118 (1960).

4 Akiyoshi K, Deguchi S, Moriguchi N, Yamaguchi S, Sunamoto J. Self-aggregates of hydrophobized polysaccharides in water. Formation and characteristics of nanoparticles. Macromolecules 26(12), 3062-3068 (1993).

5 Blackburn WH, Dickerson EB, Smith MH, Mcdonald JF, Lyon LA. Peptide-functionalized nanogels for targeted siRNA delivery. Bioconjug. Chem. 20(5), 960-968 (2009).

6 Tamura A, Oishi M, Nagasaki Y. Enhanced cytoplasmic delivery of siRNA using a stabilized polyion complex based on PEGylated nanogels with a cross-linked polyamine structure. Biomacromolecules 10(7), 1818-1827 (2009).

7 Raemdonck K, Naeye B, Buyens K et al. Biodegradable dextran nanogels for RNA interference: focusing on endosomal escape and intracellular siRNA delivery. Adv. Funct. Mater. 19(9), 1406-1415 (2009).

8 Sunasee R, Wattanaarsakit P, Ahmed M, Lollmahomed FB, Narain R. Biodegradable and nontoxic nanogels as nonviral gene delivery systems. Bioconjug. Chem. 23(9), 1925-1933 (2012).

9 Wu W, Shen J, Banerjee P, Zhou S. Core-shell hybrid nanogels for integration of optical temperature-sensing, targeted tumor cell imaging, and combined chemo-photothermal treatment. Biomaterials 31(29), 7555-7566 (2010). 
10 Wu W, Mitra N, Yan ECY, Zhou S. Multifunctional hybrid nanogel for integration of optical glucose sensing and self-regulated insulin release at physiological pH. ACS Nano 4(8), 4831-4839 (2010).

11 Zhu H, Li Y, Qiu R, Shi L, Wu W, Zhou S. Responsive fluorescent Bi2O3@PVA hybrid nanogels for temperature-sensing, dual-modal imaging, and drug delivery. Biomaterials 33(10), 3058-3069 (2012).

12 Peng H-S, Stolwijk JA, Sun L-N, Wegener J, Wolfbeis OS. A nanogel for ratiometric fluorescent sensing of intracellular $\mathrm{pH}$ values. Angew. Chem. Int. Ed. 49(25), 4246-4249 (2010).

13 Xing T, Mao C, Lai B, Yan L. Synthesis of disulfide-cross-linked polypeptide nanogel conjugated with a near-infrared fluorescence probe for direct imaging of reduction-induced drug release. ACS Appl. Mater. Interfaces 4(10), 5662-5672 (2012).

14 Hasegawa U, Nomura S-IM, Kaul SC, Hirano T, Akiyoshi K. Nanogel-quantum dot hybrid nanoparticles for live cell imaging. Biochem. Biophys. Res. Commun. 331(4), 917-921 (2005).

15 Wu W, Shen J, Banerjee P, Zhou S. Core-shell hybrid nanogels for integration of optical temperature-sensing, targeted tumor cell imaging, and combined chemo-photothermal treatment. Biomaterials 31(29), 7555-7566 (2010).

16 Oishi M, Tamura A, Nakamura T, Nagasaki Y. A smart nanoprobe based on fluorescence-quenching PEGylated nanogels containing gold nanoparticles for monitoring the response to cancer therapy. Adv. Funct. Mater. 19(6), 827-834 (2009).

17 Zhao C, Chen Q, Patel K et al. Synthesis and characterization of pH-sensitive poly(N-2-hydroxyethyl acrylamide)-acrylic acid (poly(HEAA/AA)) nanogels with antifouling protection for controlled release. Soft Matter 8(30), 7848-7857 (2012).

18 Bridges AW, Singh N, Burns KL, Babensee JE, Andrew Lyon L, García AJ. Reduced acute inflammatory responses to microgel conformal coatings. Biomaterials 29(35), 4605-4615 (2008).

19 Fujioka-Kobayashi M, Ota MS, Shimoda A et al. Cholesteryl group- and acryloyl group-bearing pullulan nanogel to deliver BMP2 and FGF18 for bone tissue engineering. Biomaterials 33(30), 7613-7620 (2012).

20 Steinhilber D, Rossow T, Wedepohl S, Paulus F, Seiffert S, Haag R. A microgel construction kit for bioorthogonal encapsulation and pH-controlled release of living cells. Angew. Chem. Int. Ed. 52(51), 13538-13543 (2013).

21 Xia Y, He X, Cao M et al. Thermoresponsive microgel films for harvesting cells and cell sheets. Biomacromolecules 14(10), 3615-3625 (2013).

22 Park KM, Lee Y, Son JY, Oh DH, Lee JS, Park KD. Synthesis and characterizations of in situ cross-linkable gelatin and 4-arm-PPO-PEO hybrid hydrogels via enzymatic reaction for tissue regenerative medicine. Biomacromolecules 13(3), 604-611 (2012).

23 Soni KS, Desale SS, Bronich TK. Nanogels: an overview of properties, biomedical applications and obstacles to clinical translation. $J$. Control. Release 240, 109-126 (2016).

24 Li J, Mooney DJ. Designing hydrogels for controlled drug delivery. Nat. Rev. Mater. 1(12), 16071 (2016).

- Guidelines for rational design of hydrogels for controlled drug delivery.

25 Ahmed EM. Hydrogel: preparation, characterization, and applications: a review. J. Adv. Res. 6(2), 105-121 (2015).

26 Zhang X, Malhotra S, Molina M, Haag R. Micro- and nanogels with labile crosslinks - from synthesis to biomedical applications. Chem. Soc. Rev. 44(7), 1948-1973 (2015).

- Emphasizes the importance of degradable linkage in the gel network.

27 Rodell CB, Mealy JE, Burdick JA. Supramolecular guest-host interactions for the preparation of biomedical materials. Bioconjug. Chem. 26(12), 2279-2289 (2015).

28 Akhtar MF, Hanif M, Ranjha NM. Methods of synthesis of hydrogels ... a review. Saudi Pharm. J. 24(5), 554-559 (2016).

29 Wu C, Strehmel C, Achazi K et al. Enzymatically cross-linked hyperbranched polyglycerol hydrogels as scaffolds for living cells. Biomacromolecules 15(11), 3881-3890 (2014).

30 Kurisawa M, Chung JE, Yang YY, Gao SJ, Uyama H. Injectable biodegradable hydrogels composed of hyaluronic acid-tyramine conjugates for drug delivery and tissue engineering. Chem. Commun. (34), 4312-4314 (2005).

31 Jin R, Hiemstra C, Zhong Z, Feijen J. Enzyme-mediated fast in situ formation of hydrogels from dextran-tyramine conjugates. Biomaterials 28(18), 2791-2800 (2007).

32 Menzies DJ, Cameron A, Munro T, Wolvetang E, Grøndahl L, Cooper-White JJ. Tailorable cell culture platforms from enzymatically cross-linked multifunctional poly(ethylene glycol)-based hydrogels. Biomacromolecules 14(2), 413-423 (2013).

33 Wu C, Bottcher C, Haag R. Enzymatically crosslinked dendritic polyglycerol nanogels for encapsulation of catalytically active proteins. Soft Matter 11(5), 972-980 (2015).

34 Zabihi F, Koeppe H, Achazi K, Hedtrich S, Haag R. One-pot synthesis of poly(glycerol-co-succinic acid) nanogels for dermal delivery. Biomacromolecules 20(5), 1867-1875 (2019).

- A straightforward one-pot enzymatic synthesis of biodegradable nanogels.

35 Moreira Teixeira LS, Feijen J, Van Blitterswijk CA, Dijkstra PJ, Karperien M. Enzyme-catalyzed crosslinkable hydrogels: emerging strategies for tissue engineering. Biomaterials 33(5), 1281-1290 (2012). 
36 Steinhilber D, Witting M, Zhang X et al. Surfactant free preparation of biodegradable dendritic polyglycerol nanogels by inverse nanoprecipitation for encapsulation and release of pharmaceutical biomacromolecules. J. Control. Release 169(3), 289-295 (2013).

37 Richter M, Steinhilber D, Haag R, Von Klitzing R. Visualization of real-time degradation of pH-responsive polyglycerol nanogels via atomic force microscopy. Macromol. Rapid Commun. 35(23), 2018-2022 (2014).

38 Dey P, Schneider T, Chiappisi L, Gradzielski M, Schulze-Tanzil G, Haag R. Mimicking of chondrocyte microenvironment using in situ forming dendritic polyglycerol sulfate-based synthetic polyanionic hydrogels. Macromol. Biosci. 16(4), 580-590 (2016).

39 Dernedde J, Rausch A, Weinhart M et al. Dendritic polyglycerol sulfates as multivalent inhibitors of inflammation. Proc. Natl Acad. Sci. USA 107(46), 19679 (2010).

40 Kuhne M, John T, El-Sayed K et al. Characterization of auricular chondrocytes and auricular/articular chondrocyte co-cultures in terms of an application in articular cartilage repair. Int. J. Mol. Med. 25(5), 701-708 (2010).

41 Schlaich C, Fan Y, Dey P et al. Universal, surfactant-free preparation of hydrogel beads on superamphiphobic and slippery surfaces. Adv. Mater. Interfaces 5(7), 1701536 (2018).

42 Dey P, Hemmati-Sadeghi S, Haag R. Hydrolytically degradable, dendritic polyglycerol sulfate based injectable hydrogels using strain promoted azide-alkyne cycloaddition reaction. Polym. Chem. 7(2), 375-383 (2016).

- Copper-free click as bioorthogonal approach for hydrogel synthesis.

43 Sridhar BV, Brock JL, Silver JS, Leight JL, Randolph MA, Anseth KS. Development of a cellularly degradable PEG hydrogel to promote articular cartilage extracellular matrix deposition. Adv. Healthcare Mater. 4(5), 702-713 (2015).

44 Dadsetan M, Szatkowski JP, Yaszemski MJ, Lu L. Characterization of photo-cross-linked oligo[poly(ethylene glycol) fumarate] hydrogels for cartilage tissue engineering. Biomacromolecules 8(5), 1702-1709 (2007).

45 Roberts JJ, Bryant SJ. Comparison of photopolymerizable thiol-ene PEG and acrylate-based PEG hydrogels for cartilage development. Biomaterials 34(38), 9969-9979 (2013).

46 Zhang C, Sangaj N, Hwang Y, Phadke A, Chang C-W, Varghese S. Oligo(trimethylene carbonate)-poly(ethylene glycol)-oligo(trimethylene carbonate) triblock-based hydrogels for cartilage tissue engineering. Acta Biomater. 7(9), 3362-3369 (2011).

47 Von Lospichl B, Hemmati-Sadeghi S, Dey P et al. Injectable hydrogels for treatment of osteoarthritis - a rheological study. Colloids Surf. B Biointerfaces 159, 477-483 (2017).

48 Hemmati-Sadeghi S, Dey P, Ringe J, Haag R, Sittinger M, Dehne T. Biomimetic sulfated polyethylene glycol hydrogel inhibits proteoglycan loss and tumor necrosis factor- $\alpha$-induced expression pattern in an osteoarthritis in vitro model. J. Biomed. Mater. Res. $B$ Appl. Biomater. 107(3), 490-500 (2019).

49 Herrmann A, Kaufmann L, Dey P, Haag R, Schedler U. Bioorthogonal in situ hydrogels based on polyether polyols for new biosensor materials with high sensitivity. ACS Appl. Mater. Inter. 10(13), 11382-11390 (2018).

50 Herrmann A, Rödiger S, Schmidt C, Schierack P, Schedler U. Spatial separation of microbeads into detection levels by a bioorthogonal porous hydrogel for size-selective analysis and increased multiplexity. Anal. Chem. 91(13), 8484-8491 (2019).

51 Randriantsilefisoa R, Cuellar-Camacho JL, Chowdhury MS, Dey P, Schedler U, Haag R. Highly sensitive detection of antibodies in a soft bioactive three-dimensional bioorthogonal hydrogel. J. Mater. Chem. B 7(20), 3220-3231 (2019).

- Represents an alternative method to commonly used immunoassays.

52 Heinrichs V, Dieluweit S, Stellbrink J et al. Chemically defined, ultrasoft PDMS elastomers with selectable elasticity for mechanobiology. PLoS ONE 13(4), e0195180 (2018).

53 Randriantsilefisoa R, Hou Y, Pan Y et al. Interaction of human mesenchymal stem cells with soft nanocomposite hydrogels based on polyethylene glycol and dendritic polyglycerol. Adv. Funct. Mater. 30(1), 1905200 (2020).

54 Wang X, Li S, Yan C, Liu P, Ding J. Fabrication of RGD micro/nanopattern and corresponding study of stem cell differentiation. Nano Lett. 15(3), 1457-1467 (2015).

55 Randriantsilefisoa R, Nie C, Parshad B, Pan Y, Bhatia S, Haag R. Double trouble for viruses: a hydrogel nanocomposite catches the influenza virus while shrinking and changing color. Chem. Commun. 56(24), 3547-3550 (2020).

- Dual responsive hydrogels recognize virus by changing their size and color.

56 Papp I, Sieben C, Ludwig K et al. Inhibition of influenza virus infection by multivalent sialic-acid-functionalized gold nanoparticles. Small 6(24), 2900-2906 (2010).

57 Miyata T, Jige M, Nakaminami T, Uragami T. Tumor marker-responsive behavior of gels prepared by biomolecular imprinting. Proc. Natl Acad. Sci USA 103(5), 1190 (2006).

58 Dey P, Bergmann T, Cuellar-Camacho JL et al. Multivalent flexible nanogels exhibit broad-spectrum antiviral activity by blocking virus entry. ACS Nano 12(7), 6429-6442 (2018).

59 Bhatia S, Hilsch M, Cuellar Camacho JL et al. Adaptive flexible sialylated nanogels as highly potent influenza A virus inhibitors. Angew. Chem. Int. Ed. 59, 12417-12422 (2020).

- Highlights the importance of flexible nanogels. 
60 Zabihi F, Wieczorek S, Dimde M, Hedtrich S, Börner HG, Haag R. Intradermal drug delivery by nanogel-peptide conjugates; specific and efficient transport of temoporfin. J. Control. Release 242, 35-41 (2016).

61 Dimde M, Neumann F, Reisbeck F et al. Defined $\mathrm{pH}$-sensitive nanogels as gene delivery platform for siRNA mediated in vitro gene silencing. Biomater. Sci. 5(11), 2328-2336 (2017).

62 Dimde M, Sahle FF, Wycisk V et al. Synthesis and validation of functional nanogels as $\mathrm{pH}$-sensors in the hair follicle. Macromol. Biosci. 17(10), 1600505 (2017).

63 Obst K, Yealland G, Balzus B et al. Protein corona formation on colloidal polymeric nanoparticles and polymeric nanogels: impact on cellular uptake, toxicity, immunogenicity, and drug release properties. Biomacromolecules 18(6), 1762-1771 (2017).

64 Zhang X, Achazi K, Steinhilber D, Kratz F, Dernedde J, Haag R. A facile approach for dual-responsive prodrug nanogels based on dendritic polyglycerols with minimal leaching. J. Control. Release 174, 209-216 (2014).

65 Zhang X, Achazi K, Haag R. Boronate cross-linked ATP-and pH-responsive nanogels for intracellular delivery of anticancer drugs. $A d v$. Healthcare Mater. 4(4), 585-592 (2015).

66 Zhang X, Zhang K, Haag R. Multi-stage, charge conversional, stimuli-responsive nanogels for therapeutic protein delivery. Biomater. Sci. 3(11), 1487-1496 (2015).

67 Steinhilber D, Sisson AL, Mangoldt D, Welker P, Licha K, Haag R. Synthesis, reductive cleavage, and cellular interaction studies of biodegradable, polyglycerol nanogels. Adv. Funct. Mater. 20(23), 4133-4138 (2010).

68 Zhou H, Steinhilber D, Schlaad H, Sisson AL, Haag R. Glycerol based polyether-nanogels with tunable properties via acid-catalyzed epoxide-opening in miniemulsion. React. Funct. Polym. 71(3), 356-361 (2011).

69 Hellmund M, Zhou H, Samsonova O, Welker P, Kissel T, Haag R. Functionalized polyglycerol amine nanogels as nanocarriers for DNA. Macromol. Biosci. 14(9), 1215-1221 (2014).

70 Gao L, Zabihi F, Ehrmann S, Hedtrich S, Haag R. Supramolecular nanogels fabricated via host-guest molecular recognition as penetration enhancer for dermal drug delivery. J. Control. Release 300, 64-72 (2019).

- Produces nanogel via host-guest interactions as cross-linking strategy.

71 Oehrl A, Schötz S, Haag R. Systematic screening of different polyglycerin-based dienophile macromonomers for efficient nanogel formation through IEDDA inverse nanoprecipitation. Macromol. Rapid Commun. 41(1), 1900510 (2020).

72 Stolzer L, Vigovskaya A, Barner-Kowollik C, Fruk L. A self-reporting tetrazole-based linker for the biofunctionalization of gold nanorods. Chem. Eur. J. 21(41), 14309-14313 (2015).

73 Stolzer L, Quick AS, Abt D et al. Photo-induced surface encoding of gold nanoparticles. Chem. Commun. 51(16), 3363-3366 (2015). 